UCRL-IC-124081

$$
\text { CONF-960569-1/ M96050419 }
$$

\title{
A Review of ELMs in Divertor Tokamaks
}

D. N. Hill

This paper was prepared for submittal to the

$$
\begin{gathered}
\text { CEDIED } \\
\text { SEP } 131095 \\
\text { OSTI }
\end{gathered}
$$

12th International Conference on Plasma Surface Interactions

in Controlled Fusion Devices,

Saint Raphael, France

May 20-24, 1996

May 23, 1996

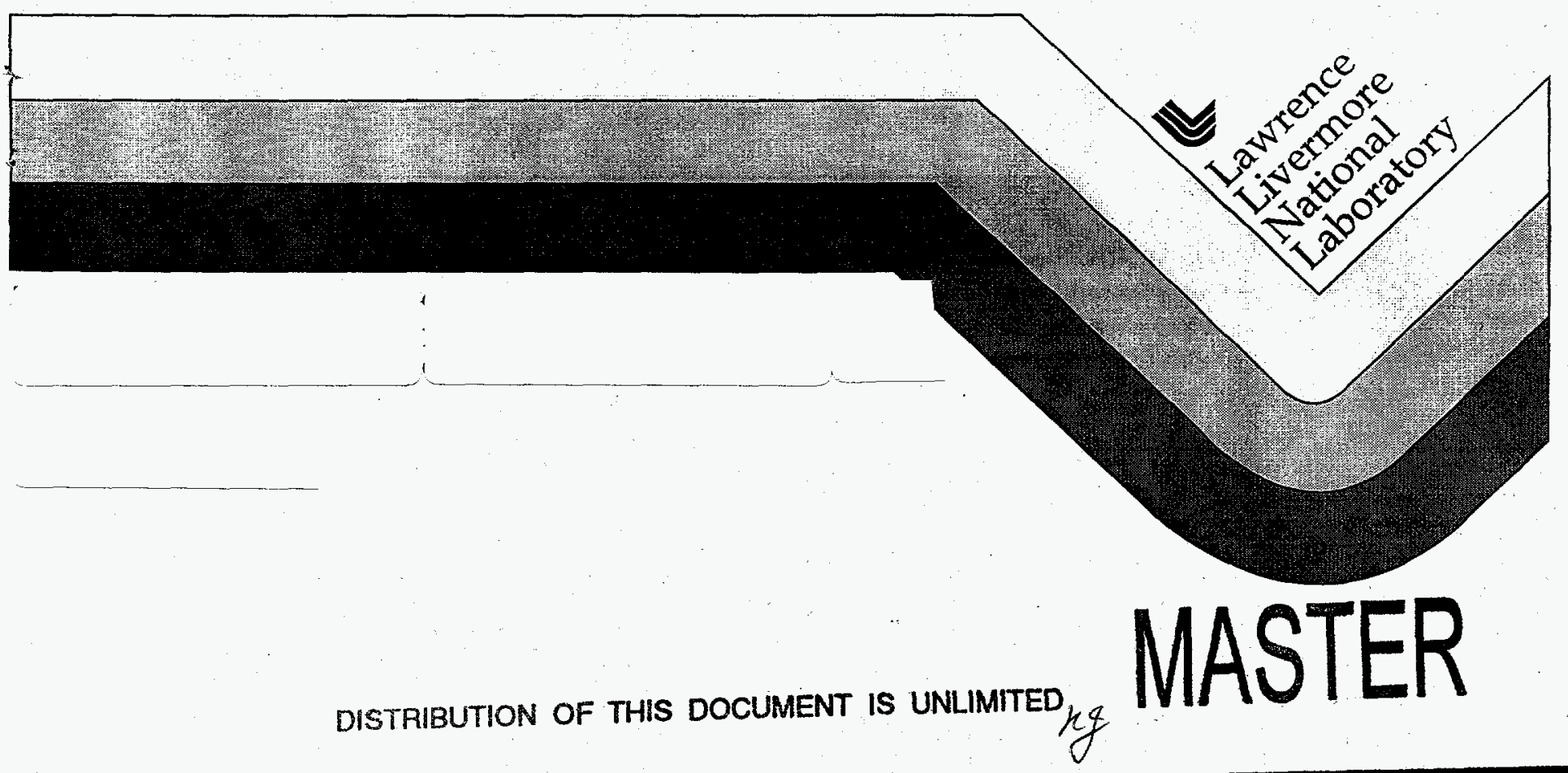




\section{DISCLAIMER}

This document was prepared as an account of work sponsored by an agency of the United States Government. Neither the United States Government nor the University of California nor any of their employees, makes any warranty, express or implied, or assumes any legal liability or responsibility for the accuracy, completeness, or usefulness of any information, apparatus, product, or process disclosed, or represents that its use would not infringe privately owned rights. Reference herein to any specific commercial product, process, or service by trade name, trademark, manufacturer, or otherwise, does not necessarily constitute or imply its endorsement, recommendation, or favoring by the United States Government or the University of California. The views and opinions of authors expressed herein do not necessarily state or reflect those of the United States Government or the University of California, and shall not be used for advertising or product endorsement purposes. 


\section{DISCLAIMER}

Portions of this document may be illegible in electronic image products. Images are produced from the best available original document. 



\title{
A Review of ELMs in Divertor Tokamaks
}

\author{
D.N. Hill
}

Lawrence Livermore National Laboratory, P.O. Box 808, Livermore, CA 94550

\section{Abstract}

Edge Localized Modes (ELMs) are becoming the focus of increasing attention by the edge physics community because of the potential impact that the large divertor heat pulses due to ELMs would have on the divertor design of future high power tokamaks such as ITER. This paper reviews what is known about ELMs, with an emphasis on their effect on the scrape-off layer and divertor plasmas. ELM effects have been measured in the ASDEX-U, C-Mod, COMPASS-D, DIII-D, JET, JFT-2M, JT$60 \mathrm{U}$, and TCV tokamaks and are reported here. At least three types of ELMs have been identified and their salient features determined. Type-1 giant ELMs can cause the sudden loss of up to $10-15 \%$ of the plasma stored energy, but their amplitude $(\Delta \mathrm{W} / \mathrm{W})$ does not increase with heating power. Type-3 ELMs are observed near the $\mathrm{H}$-mode power threshold and produce small energy dumps (1-3\% of the stored energy). All ELMs increase the scrape-off layer plasma and produce particle fluxes on the divertor targets which are as much as ten times larger than the quiescent phase between ELMs. The divertor heat pulse is largest on the inner target, unlike that of L-mode or quiescent $\mathrm{H}$-mode; some tokamaks report radial structure in the heat flux profile which is suggestive of islands or helical structures. The power scaling of Type-1 ELM amplitude and frequency has been measured in several tokamaks and has recently been applied to predictions of the ELM size in ITER. Concern over the expected ELM amplitude has led to a number of experiments aimed at demonstrating active control of ELMs. Impurity gas injection with feedback control on the radiation loss in ASDEX-U suggests that a promising mode of operation (the CDH-mode) with very small Type-3 ELMs can be maintained with heating power well above the H-mode threshold, where giant Type-1 ELMs are normally observed. While ELMs have many potential negative effects, the 
beneficial effect of ELMs in providing density control and limiting the core plasma impurity content in high confinement $\mathrm{H}$-mode discharges should not be overlooked. 


\section{Introduction}

Edge Localized Modes (ELMs) are observed in tokamak H-mode discharges and produce periodic loss of particles and energy from the edge region of the confined plasma. Their most obvious signature is a short (msec) spike in $\mathrm{H}_{\alpha}$ emission from the plasma boundary, as shown by the data from an H-mode discharge in JET, Fig. 1. Interest in ELMs arises from a number of observations: i) they tend to limit energy confinement, ii) they provide density control and limit the buildup of impurities in $\mathrm{H}$-mode, iii) they broaden the scrape-off layer density profile and modulate ICRH antenna coupling, iv) they produce large heat pulses on the plasma facing components, and v) they increase sputtering of first-wall materials.

ELMs were first observed in the ASDEX tokamak operating in a double-null configuration[1], and subsequently seen in PDX[2] (also double null), Doublet-III[3] (single null) and other single-null divertor tokamaks, and finally even in limiter tokamaks once $\mathrm{H}$-mode had been achieved in that configuration[4]. Since their discovery, considerable effort has been devoted to identifying the underlying instabilities that produce ELMs, which look very much like a relaxation phenomena due to the sawtooth-like behavior of the edge density and temperature (e.g., see Fig. 1(c)). So far, at least three types of ELMs have been identified[5], and it appears that each is due to a different instability. Type-1, or Giant ELMs, have for some time been associated with ideal ballooning modes driven unstable by the high edge pressure gradients in H-mode. Type-2, or Grassy ELMs, are seen only in highly shaped discharges which have access to the second-stable ballooning region near the edge[6]. Type-3 ELMs are observed when the power crossing the separatrix is just above the $\mathrm{H}$-mode power threshold, and may result from resistive instabilities, since they occur at pressure gradients well below the ideal limit and can be stabilized by increasing the edge electron temperature.

Individual ELMs produce a significant perturbation on the scrape-off layer (SOL) plasma. During the early part of the ELM (the first $100 \mu \mathrm{sec}$ or so) magnetic $[7,8]$ and electrostatic[9] turbulence is high and plasma inside the separatrix is quickly lost via radial transport[10]. Outside the separatrix the electron temperature rises briefly, but the high parallel electron thermal conductivity in the SOL limits the duration of the temperature pulse to the time when turbulent radial energy transport is high. The density in the SOL also increases during the ELM and the profile broadens by factors of five or more. This perturbation generally lasts longer than the temperature excursion because of slower parallel particle transport and finite recycling at the 
divertor targets; thus the $\mathrm{H}_{\alpha}$ pulse associated with the ELM generally lasts longer than the heat pulse on the divertor targets[11, 12]. One feature of ELMs which is not understood is why they produce a larger heat pulse at the inner divertor target than at the outer target plates in single-null divertors when the usual thermal conduction in between ELMs is peaked at the outer target plates[13, 14].

The effect of ELMs on the edge, scrape-off layer, and divertor plasmas is a strong function of their amplitude and frequency, which are usually coupled. That is, generally, the more rapid the ELM rate, the smaller the amplitude of the individual ELMs. Isolated Giant ELMs occur at repetition rates of $10 \mathrm{~Hz}$ or less can produce a loss of as much as $10-15 \%$ of the stored energy and density in the confined plasma in a few milliseconds and can effect the density and temperature profiles in to as far as half the minor radius of the plasma. While not significant in present machines, the large burst of heat and particles associated with such ELMs could damage the plasma facing components in future high power devices such as the proposed ITER[15]. At the other extreme, the individual effects of very rapid (100's $\mathrm{Hz}$ to $\mathrm{kHz}$ repetition rates) Grassy Type-2 or Type-3 ELMs may be too small to measure directly and can only be inferred from time-averaged measurements.

Because of the large variability in ELM size and their impact on confinement, as well as the potential for ELM-related damage in future devices, a number of scaling studies are now underway. So far, several machines have mapped out their operating space for obtaining different types of ELMs; ASDEX-U for example[16, 17], finds that Type-3 ELMs only occur in a narrow window of $\mathrm{P}_{\mathrm{H}}<\mathrm{P}_{\text {sep }}<1.2 \mathrm{P}_{\mathrm{H}}$, where $\mathrm{P}_{\mathrm{H}}$ is the $\mathrm{H}$-mode power threshold and $\mathrm{P}_{\text {sep }}$ is the net heating power crossing the separatrix. In some cases data has been obtained showing how ELM amplitude and frequency vary with plasma current, heating power, or edge temperature, but no scaling relations have been developed that allow for reliable prediction of the amplitude and frequency of individual ELMs in a given discharge. Comparisons relating ELM amplitude and frequency on different machines is just beginning; very preliminary initial results suggest that the amplitude $(\Delta W / W)$ of Type-1 ELMs decreases as $P_{\text {sep }} / P_{H} A$, where $A$ is the plasma surface area[18]. However, much more work of this type is needed and is planned under the auspices of the ITER project.

Ultimately, active control of ELM frequency and amplitude is desired. Experiments have shown that it is possible to produce ELMs in otherwise ELM-free $\mathrm{H}$-mode discharges, or change their frequency over a limited range using edge heating or magnetic perturbations. Plasma shaping has also been used to control 
ELMs[6, 19]; however, many of these experiments were carried out in low power discharges where the ELMs were rather small. In higher power discharges, ASDEX$U$ has managed to use edge radiation to lower the power flow across the separatrix $[20,21]$ so as to operate near the $\mathrm{H}$-mode power threshold and obtain small amplitude, high frequency Type-3 ELMs, instead of Type-1 ELMs. It remains, however, to show that small amplitude ELMs can be produced when operating well above the H-mode power threshold. Hopefully, that identification of the modes responsible for ELMs will guide efforts to control them.

In the remainder of this review paper, we will discuss further details of ELM characteristics and their effects in divertor tokamaks. In Section 2 we expand on the differences between the various types of ELMs and on the operating space occupied by each. Section 3 focuses on the effect of ELMs on the scrape-off layer and divertor plasma, while Section 4 covers the ELM effects on the divertor plasma. Data showing the scaling of ELM frequency and amplitude and extrapolation to ITER appears in Section 5. Section 6 briefly reviews results of experiments aimed at ELM control. Finally, we conclude with a discussion of the implications of our present understanding and suggest directions that future work should take. Note that in this paper we focus on SOL and divertor plasma effects and do not include much discussion in this paper on measurements of the magnetic precursors or the possible identification of various instabilities as the cause of particular types of ELMs. This subject has been covered in other recent reviews[22]. We also restrict this discussion to ELMs in divertor tokamaks since the common features of ELMs in limiter discharges are mainly related to the stability questions which we do not have space to address here.

\section{Overview of ELM behavior}

All tokamaks which have achieved $\mathrm{H}$-mode operation have reported observing ELM activity regardless of magnetic configuration (inside, outside limiter, single and double null) or heating method (ohmic, RF, or neutral beam). Table 1 shows the types of ELM behavior reported for presently operating divertor tokamaks.

One factor which does seem to impact ELM behavior in various machines is the divertor configuration. For example, when the baffling of the ASDEX divertor was tightened at the end of its operating life, ELM-free H-mode was much more difficult to obtain and steady ELM activity was the usual situation[23]. Similarly, in JET, 
when the more tightly baffled Mk1 divertor was installed, ELMy H-modes became more typical and long ELM-free periods were not so common[24]. One might speculate that these changes resulted from having a lower edge particle source with the better gas baffling, which in turn produced higher edge temperatures that would tend to stabilize the Type-3 ELMs found in those machines at that time.

Type-1 Giant ELMs and Type-3 ELMs are now the most commonly observed in divertor tokamaks, and will be described more fully below. Type-2 "grassy" ELMs have been reported only in discharges with a high degree of shaping (elongation and/or triangularity) $[6,19]$. Usage of the term "grassy" to describe ELM activity should be clarified, as it is sometimes applied rather loosely in the literature. Many times, it is difficult to know for sure what kind of ELM activity is present from a casual inspection of the $\mathrm{H}_{\alpha}$ trace alone, since the amplitude and frequency of any individual type of ELM can vary considerably. In the case of very rapid ELMs the $\mathrm{H}_{\alpha}$ trace can be very irregular and look quite "grassy." Unfortunately, this term was applied to rapid ELMs and came into popular usage before the more subtle differences in ELM physics were appreciated and the three categories of ELMs identified. In this article we will use "grassy ELMs" only when referring to Type-2 ELMs, even though many times the $\mathrm{H}_{\alpha}$ trace showing Type-3 ELMs also may look "grassy." Recently, Weisen has observed ELMs on TCV which he calls "mossy" ELMs because they appear to be similar to Type-2 grassy ELMs, but positive correlation has not been made as yet.

\subsection{Type-1 Giant ELMs}

Giant ELMs are observed when the power flow through the edge plasma is well above the H-mode power threshold (about $20 \%$ in ASDEX-U, DIII-D, and JET). The giant ELMs in a JET NBI heated discharge shown in Fig. 1 are fairly typical of those observed in all machines. Shortly after the start of the heating pulse (a) the plasma makes a transition to $\mathrm{H}$-mode: the divertor $\mathrm{H}_{\alpha}$ emission drops (d) and the stored energy (b) and density (c) begin to rise. After an initial ELM-free period, giant ELMs appear as $\mathrm{H}_{\alpha}$ spikes starting at $14.7 \mathrm{sec}$ and continue until the end of the heating pulse. The amplitude of the $\mathrm{H}_{\alpha}$ spikes is much larger than either the initial level or the level in between the spikes. In between the ELMs the density and energy rise at nearly the same rate as at the initial $\mathrm{H}$-mode transition, signaling the return of the transport barrier. 
Once the ELMy period starts, the stored energy quits rising and the rate of density buildup is reduced. In general, the periodic edge losses due to ELMs produce a larger effect on the global particle balance than on the energy balance because the $\mathrm{H}$-mode density profile is flat but the pressure profile is peaked (central heating and edge fueling combined with an edge transport barrier). Direct measurements of the prompt energy loss due to ELMs[7, 11, 18] show that the time-average contribution to the energy confinement is rather modest $(10-20 \%)$, which is consistent with other comparisons between ELMy and ELM-free plasmas[25]. In some situations, the ELMs cause a return to L-mode so that energy confinement is degraded between the ELMs as well. Parail[7] has shown that for this situation in JET, the L-mode periods have a much larger effect on confinement than the ELMs themselves. Generally, ELMS affect confinement more strongly at higher beta and density more strongly at lower beta.

One very positive result of ELM activity in H-mode plasmas is that impurity buildup is generally avoided[23, 26-28] compared to ELM-free plasmas. As in the case of the main ion density, this results from the fact that the ELMs reduce the particle confinement time by increasing the radial transport (e.g., see [28]) in the edge plasma where the impurity ion source is located. Time-average impurity transport is further enhanced if the ELM produces a transition back to L-mode. Almost all the ELM-induced edge transport is outward since the gradients are steepest on the separatrix, or if, as some have suggested, during the ELM itself the outward radial transport is mostly convective[10, 29]. With central plasma fueling from neutral beam injection in DIII-D, the onset of ELMs has been observed to lower the central impurity ion concentration following an initial buildup during the ELM-free period after the H-mode transition. However, the ELMs apparently do not directly affect impurity transport in the core, where the background neoclassical effects dominate.

The ELM itself is a relatively fast MHD event $(100-200 \mu \mathrm{sec})$ which is characterized by magnetic and electrostatic turbulence. No magnetic precursors to Type-1 ELMs are observed[22], though precursor fluctuations in the edge electron temperature have been reported on JET[30, 31]. During the time of the strong fluctuations the electron density inside the separatrix (in to about $r / a=0.9$ ) drops significantly; as shown in Fig. 2. In DIII-D little change in electron temperature is observed in the edge region with Thomson scattering, while ECE data from JET[7, 28] indicate that large ELMs $(\triangle W / W \geq 10 \%)$ can abruptly reduce the edge $T_{e}$ by as much as $40 \%$. Edge ion temperature seems unaffected by ELMs[32, 33], though the temporal resolution of present ion temperature diagnostics (charge-exchange 
recombination spectroscopy) is marginal. Mahdavi[10] has concluded that, because the electron temperature remains high in the affected region of DIII-D, the energy is lost by turbulent radial diffusion or convection rather than island formation producing a channel for conduction losses along field lines, since parallel conduction would rapidly lower the electron temperature. Other authors have argued for diffusion by magnetic fluctuations based on analysis of Mirnov activity during the ELM[34].

It is well known that the frequency of Type-1 ELMs increases with heating power, and this is one of the main indicators used to identify them[35]. More careful measurements and experiments with impurity gas puffing have shown that it is the power transported through the edge plasma which is the independent variable that governs the ELM behavior rather than simply the total heating power. Increasing the edge particle source has also been shown to increase Type-1 ELM frequency. In addition, both DIII-D[35] and JET[36] have reported that the ELM frequency decreases strongly with plasma current, as shown in Fig. 3.

The effect of heating power, particle source strength, and plasma current on ELM frequency are consistent with the picture that Type-1 ELMs result when the edge pressure gradient exceeds the ideal ballooning mode limit. This picture was developed following careful measurements in DIII-D by Gohil, et al., of the buildup of the edge electron pressure gradient prior to an ELM[37]. The critical pressure gradient for the destabilization of the ideal ballooning mode[6] depends on the local flux-surface averaged magnetic shear, $S$, and the safety factor, $q$, which Gohil evaluated at the $95 \%$ flux surface in his study $\left(\mathrm{S}_{95} / \mathrm{q}_{95}^{2}\right)$. Thus, anything which increases the rate that the pressure builds up reduces the time required to hit the stability limit and increases the ELM frequency, while anything that raises the limit, such as increasing the current (lowers $q_{95}$ ) or triangularity (increases $\mathrm{S}$ ), increases the time required to hit the stability limit and reduces the ELM frequency. Strictly speaking, this model only explains the onset of the first ELM following the H-mode transition; using it to explain the ELM frequency in stationary $\mathrm{H}$-modes, requires some assumption about how much the pressure gradient drops before edge confinement is restored.

\subsection{Type-3 ELMs}

Type-3 ELMs occur when the power through the edge plasma is just above the H-mode power threshold[22]. Their repetition rate is typically higher than that of 
Type-1 ELMs (100's $\mathrm{Hz}$ to $\mathrm{kHz}$ ) and their amplitude much smaller ( $\triangle \mathrm{W} / \mathrm{W}$ typically $1 \%$ or less). The outstanding characteristic which distinguishes Type-3 ELMs from Type- 1 is that their frequency decreases with power instead of increasing[5]. This behavior is shown in Fig. 4, which contains data from a DIII-D discharge in which the neutral beam power was steadily increased. The Type-3 ELMs are visible in the divertor $\mathrm{H}_{\alpha}$ trace (d) following the $\mathrm{H}$-mode transition at $1.745 \mathrm{sec}$ and, like the Type1 ELMs in Fig. 1, the spikes are larger than the initial $\mathrm{H}_{\alpha}$ level before the $\mathrm{H}$-mode transition. Note that the effect of the individual ELMs is not visible in the lineaverage density or stored energy traces. As the beam power was increased, the frequency of ELMs decreased as shown in (e). Later in the discharge, after a further increase in beam power, the Type- 3 ELMs were stabilized at about $3.25 \mathrm{sec}$ and replaced by Type-1 ELMs.

Detailed measurements of the edge pressure gradient show that it is well below the ballooning limit at the onset of Type-3 ELMs, thus suggesting that a different instability is operative. Magnetic precursors are observed during these ELMs[38], unlike the case of giant ELMs. ECE measurements on JET[31] clearly show that the frequency drops with increasing edge electron temperature or electron pressure gradient, thus suggesting that some sort of resistive MHD mode may be the origin of these ELMs. In fact, the variation of ELM frequency with power shown in Fig. 4(e) is very similar to that of $f$ vs. $T_{e}$ shown by Colton. Alternatively, it may be that the threshold for Type-3 ELMs is tied to the H-mode power threshold rather than simply $T_{e}$, as suggested by recent experiments on DIII-D in which the H-mode threshold was varied independently of edge electron temperature by varying the toroidal field strength at constant density and heating power[18, 39].

\subsection{Type-2 and others}

Other types of ELMs also exist. Type-2 "grassy" ELMs have been observed in highly shaped plasmas in which the ballooning stability parameter $\left(\mathrm{S}_{95} / \mathrm{q}_{95}^{2}\right)$. is small enough to allow access to the 2nd stable region. JT-60U was able to obtain such ELMs by modestly increasing the plasma triangularity. In a series of experiments in

DIII-D, Ozeki et al, changed the triangularity of the plasma so as to vary $\left(\mathrm{S}_{95} / \mathrm{q}_{95}^{2}\right)$ in a controlled manner and they were able to systematically switch from Type- 1 to Type-2 ELMs[6], as shown in Fig. 5(a). 
Virtually nothing is known about how the frequency and amplitude of Type-2 ELMs depends on power, though they are so small as to not be a problem, even while maintaining density control. Recently, similar small ELMs, called "mossy" ELMs, were observed in ohmic H-modes in TCV[19], which is also highly shaped. However, it is not known if these phenomena are the same.

At the other extreme, both JET[40] and DIII-D report observing very large ELMs during high confinement, high beta discharges; these events can affect the core plasma well inside $r / a=0.5$ and can lead to long term (long after the MHD event) confinement degradation. In DIII-D, magnetic precursors are clearly observed, distinguishing these from the usual Type-1 ELMs. In JET, some of these events cause the loss of as much as $1 \mathrm{MJ}$ of stored energy and produce large influx of impurities, presumably due to sputtering or damage of plasma facing components[40]. Transport analysis of the effect of these events show that the degradation of confinement results from a long L-mode period of up to $200 \mathrm{msec}$ (see Fig. 5(c)) following the initial collapse[7].

Finally, we should mention that some $\mathrm{H}_{\alpha}$ noise may look like ELMs, but is actually a dithering between L-mode and H-mode[41], as in Fig. 5(d). This dithering is usually observed just prior to a clear $\mathrm{H}$-mode transition or at the end of the $\mathrm{H}$ mode just before the plasma returns to L-mode or ohmic confinement. Such dithering has been explained as the result of the initial density rise increasing the $\mathrm{H}$ mode power threshold to the point where a back transition occurs, which in turn reduces the density and the power threshold, allowing the cycle to repeat again[22].

\subsection{Operating Space}

While ELM behavior can vary considerably from machine to machine, it is generally possible to map out reproducibly the operating space for obtaining each kind of ELM in a given tokamak. The easiest way to do this is to vary the power crossing the separatrix; data from ASDEX-U in Fig 6 show how steadily increasing the beam heating power first produces an H-mode with small Type-3 ELMs, which then disappear to be replaced by Type-1 ELMs of steadily increasing frequency[41]. Similar reproducible behavior is observed in the other divertor tokamaks with $\mathrm{H}$ mode.

The reproducibility of ELM characteristics in a given tokamak makes it possible to put together a database from a number of discharges so that the operating space 
for each type of ELM can be quantified. Such data from ASDEX-U appear in Fig. 7, where the operating space is characterized by the ratio of the heating power to the $\mathrm{H}$ mode threshold power $\left[\mathrm{P}_{\mathrm{sep}} / \mathrm{A}\left(\mathrm{MW} / \mathrm{m}^{2}\right)=0.044 \mathrm{n}_{\mathrm{e}} \mathrm{B}_{\mathrm{t}}\left(10^{20} \mathrm{~m}^{-3} \mathrm{~T}\right)\right][17]$, where $\mathrm{n}_{\mathrm{e}}$ is the line-average density and $\mathrm{B}_{\mathrm{t}}$ the toroidal field. Small Type-3 ELMs appear only near the power threshold, while Type-1 ELMs require heating power more than $20 \%$ above the H-mode threshold; in between lies ELM-free operation. Note that the boundary between the ELM-free and Type-3 ELMing regions is not sharply defined and probably depends on parameters which are hard to quantify, such as wall conditions or divertor geometry. Reliable operation in the region with Type-3 ELMs therefore usually requires iteration or some sort of feedback control for a given set of wall conditions. Also, since the character of the ELMs can change so dramatically with only a small change in power above the $\mathrm{H}$-mode threshold $( \pm 10 \%$ goes from Type-1 to ELM-free to Type-3), we can see that it is difficult to apply the experience from one machine to predict, a priori, the ELM behavior in another device, given the large error bars in the $\mathrm{H}$-mode power threshold (at least $\pm 50 \%$ ). Therefore, it is not possible to use data such as that in Fig. 7, to predict the ELM characteristics in ITER with a great deal of confidence.

The effect of divertor geometry on the operating space for ELMs is not understood and remains a significant ${ }^{\dagger}$ uncertainty in comparing ELM data from different tokamaks. Both ASDEX and JET have made significant changes to their divertor geometry and neutral gas baffling and have seen large changes in the ELMs. In JET with its original open divertor, most $\mathrm{H}$-mode discharges were ELM-free; now with the internal coils and pumped side walls, ELMy $\mathrm{H}$-modes are routinely observed[24]. It is not so clear whether the change resulted from a change in edge ballooning stability due to the new plasma shape or from changed neutral recycling due to the new divertor shape. In ASDEX, the volume of the divertor chamber was reduced significantly when water cooled target plates were installed. Subsequently, ELM-free $\mathrm{H}$-modes were much harder to obtain, ELMy $\mathrm{H}$-modes had higher ELM frequencies, and confinement was degraded[42, 43]. Good H-mode performance was restored when gas leaks from the new divertor were closed and boronization was used to further reduce recycling. Again, in this case no correlation between measurable parameters changed by the geometry and ELM characteristics was established. Thus, we cannot in a quantitative way describe how divertor geometry or wall conditioning affect the operating space for ELMs. Perhaps, as more tokamaks modify their divertor geometry and divertor models can reproduce the observed 
changes in divertor conditions, we will be able to deduce the fundamental relationships that effect the ELM behavior.

\section{Effect of ELMs on the Edge and Scrape-off layer plasma}

Most measurements of the effect of individual ELMs on the edge and SOL plasma are obtained for Type-1 ELMs since the perturbations due to Type- 2 or 3 typically are too small to measure reliably. Thus, all data and effects covered here apply to Type- 1 ELMs unless otherwise stated. Fig. 2 shows the changes in electron density and temperature profile in the edge plasma produced by a typical giant ELM in DIII-D $(\Delta \mathrm{W} / \mathrm{W} \leq 5 \%)$ as measured by Thomson scattering. Inside the separatrix the density and its gradient drop significantly over a distance of several $\mathrm{cm}$ in less than $0.5 \mathrm{msec}$, while Te is much less affected; similar results are obtained in ASDEXU[29] using a lithium beam diagnostic for density and an ECE radiometer for temperature. In contrast, ECE measurements in JET[7, 28] show fairly large changes in Te (up to a $40 \%$ drop). Measurements of Ti also show little change[32, 33], but the temporal resolution of charge-exchange recombination spectroscopy diagnostics so far has been marginal for such measurements.

The radial distance over which the core plasma is affected increases with the size of the ELM might be expected; some machines[7,29] report effects in as far as a/2, but more usually only the outer $10-15 \%$ of the minor radius is affected. What governs the size of this region is not well understood, though data from hot-ion H-modes in JT-60U suggests that it scales as the size of the poloidal ion gyroradius[44], but this is probably not consistent with the very deep penetration of the ELMs sometimes observed. The size of this region and its scaling is a key parameter for understanding the scaling of ELM amplitude and frequency, as discussed below. Clear inward propagation of the temperature reduction away from the unstable region has been measured with ECE diagnostics in ASDEX-U[29].

The expulsion of a large fraction of the density from edge of the plasma represents a significant contribution to the time-averaged particle balance in ELMy $\mathrm{H}$-mode plasmas, and allows quasi-stationary $\mathrm{H}$-modes to be maintained. This is possible because the $\mathrm{H}$-mode density profile is very flat due to the combination of edge fueling from recycling and formation of a strong particle transport barrier just inside the separatrix [45]. Indeed, the usual density rise which is characteristic of the $\mathrm{H}$-mode transition is greatly reduced or halted entirely following the appearance of ELMs. Time-resolved measurements of the density profile allow the effect of the 
ELMs on the particle balance to be determined directly. Assuming that the perturbation on plasma density is uniform poloidally, integration of the change in the density profile before and after typical Type- 1 ELMs shows that each produces a loss of up to $\Delta N \simeq 10-15 \%$ of the total particle content [11, 22]. The time-average loss rate $\mathrm{f} \Delta \mathrm{N}$ computed in this way is found to match the initial density rise, $\mathrm{dN} / \mathrm{dt}$, thus showing that the ELMs provide the steady state density control in H-mode.

The mechanism responsible for the rapid loss of plasma from the edge during the ELM has not been clearly identified. Candidates include parallel conduction along suddenly open field lines produced by island formation, turbulent radial transport due to electrostatic or electromagnetic fluctuations, or radial convection due to large scale-length potential structures. No clear winner has emerged to explain the data from all tokamaks. Buttery[46], calculated the turbulent diffusion coefficient, $\chi_{r}$, due to the measured $m, n$ spectrum of the magnetic fluctuations associated with ELMs in COMPASS-D and concluded that it was about the right order of magnitude to explain the measured loss in stored energy. Mahdavi[10] assumed that convective radial transport was operative for DIII-D and calculated that the measured drop in edge $T_{e}$ produced by ELMs was too small to be the result of parallel conduction along field lines in magnetic islands. A similar model of outward convective transport has been proposed by O'Mullane for impurities in ASDEX-U. On the other hand, as will be shown later in Section 4, the observed in/out asymmetries and poloidal structure of ELM-induced divertor heat flux are strongly suggestive of magnetic islands. Some estimates for $D_{\perp}$ during ELMs, 5$10 \mathrm{~m}^{2} / \mathrm{sec}$ based on density profile analysis in ASDEX-U[47] and JET[7], are consistent with electrostatic turbulent transport, but no data on $\tilde{\phi}$ and $\tilde{n}$ are available to confirm this picture.

In the scrape-off layer, the plasma density rises factors of 3-5 and the density profile broadens about the same amount. Langmuir probes show that electrostatic fluctuations in the SOL increase during the ELM. Increased density is usually observed all the way to the vessel wall, and this has a direct impact on RF antenna loading (it momentarily increases). Numerical simulations of the heat and particle pulse in the SOL due to ELMs[47-49] show that the observed broadening of the SOL can be explained by an order of magnitude increase in the diffusion coefficient (from $D_{\perp}=0.55 \mathrm{~m}^{2} \mathrm{sec}^{-1}$ to $5 \mathrm{~m}^{2} \mathrm{sec}^{-1}$ and $\chi_{\mathrm{e}}$ from 0.1 to $5 \mathrm{~m}^{2} \mathrm{sec}^{-1}$ ) in the scrape-off layer. The modeling also shows that the electron temperature in the SOL drops rapidly once the turbulent radial transport out of the core (typically lasting $0.2 \mathrm{msec}$ or less) drops, while the density remains high for longer periods due to particle recycling at the 
target plates. Thus, the SOL density profile in an ELMy discharge can be broader even in between ELMs than in the corresponding ELM-free plasma. Such is the case for the ELMy H-mode discharge shown in Fig. 8. The Thomson scattering data show that the SOL density is much higher after $2.7 \mathrm{sec}$ when ELMs appear, while the SOL temperature is still low.

A broader SOL plasma should produce a beneficial screening effect against impurity influx while minimizing damage to plasma facing components. Improved screening against high- $\mathrm{Z}$ impurities in ELMy $\mathrm{H}$-mode ASDEX plasmas was identified by a slow reduction of the core impurity content after the onset of ELMs which was much larger than the fast drop observed after each ELM[23].

\section{ELM effects in the divertor}

The sudden loss of confinement from the main plasma produces a large burst of particles and energy in the scrape-off layer which is transported to the divertor region. Several characteristic time scales are involved: i) the electron thermal conduction time $(-100 \mu \mathrm{sec}$ or less), ii) the ion flow time ( $1 \mathrm{msec})$, and iii) the neutral gas transport time out of the divertor $(-2-10 \mathrm{msec})$. The energy flow to the target plates is dominated by electron conduction along field lines and so the heat pulse arrives first and lasts as long as energy is being transported across the separatrix. Ion flow from the main plasma to the divertor is limited to the ion sound speed, so the divertor density builds more slowly, though the initial heat pulse can increase ionization of neutrals and produce an initial small burst of particles. Finally, after the ions are transported to the divertor target they begin to recycle and some milliseconds are required to establish a new equilibrium.

The ELM heat pulse to the divertor targets has been measured in a number of tokamaks using infrared thermography[50-54]. The heat flux is inferred from the time history of the surface temperature using the known thermal properties of the materials (recent analysis of transient heat pulses in which negative heat flux is obtained following large transient loads has suggested that the target plates may have a thin coating with different thermal properties[52]). These measurements usually can provide a radial profile of the heat flux at one toroidal location with a temporal resolution of about $100 \mu \mathrm{sec}$, which is marginal for recovering the initial heat pulse, but is more than adequate to recover the relative timing between the rise in target-plate ion flux and $\mathrm{H}_{\alpha}$ emission. 
Peak divertor heat flux during ELMs ranges from 2-10 times or more the time average heat flux; values as high as $50-100 \mathrm{MW} / \mathrm{m} 2$ have been reported. The heat pulses in ASDEX-U are generally smaller than those in DIII-D, which are in turn, smaller than those in JT-60U. Figure 9 shows the divertor heat pulse due to Type-1 ELMs in ASDEX-U. In (a) we can see both the steady heat flux in the quiescent phase between the ELMs and the pulses due to two ELMs; the steady heat flux is largest on the outer target plate, while the ELM pulse is largest on the inner plate. These asymmetries (in between and during the ELMs) are typical of those reported on DIIID[11], JET[8], and JT-60U[55] for Type-1 ELMs. In general, the in/out asymmetry is the same for Type-3 ELMs, though some ICRH sustained H-modes in ASDEX-U showed the ELMs producing a larger pulse on the outer plate. Unlike the steady heat flux between ELMs, the in/out asymmetry during ELMs does not seem affected by a reversal of the ion $\nabla B$ drift direction, though data is very limited[55].

The heat flux profiles during ELMs show quite a variation among the different tokamaks. In ASDEX-U, the profile during the ELM is quite similar in shape to the ELM-free heat flux profile: it is peaked near the separatrix and falls off with a characteristic length which is not too different from that of the quiescent phase, as shown in Fig. 9(b) Previously it was reported[16] that the heat flux profile during ELMs was broadened considerably compared to the ELM-free value, but further analysis shows that the fall-off length near the peak is not changed, though a broad tail far out in the profile does appear. Locally, this tail produces a large relative change in heat flux, but it is very much less than at the peak near the separatrix, so it is not much cause for concern.

The relatively small change in heat flux profile is in sharp contrast with the effect of ELMs on the SOL density profile, which broadens due to an increase in $D_{\perp}$, as discussed above. Since the heat flux is determined by the rapid parallel electron conduction, while the density profile is governed by much slower ion convection and target plate recycling, it is not too surprising to find that the ELM broadens one but not the other profile.

Measurements of the heat flux profiles in other machines do not always show such well-behaved monotonic characteristics. In ASDEX, the heat flux profile at the outer targets showed clear evidence for radial structure[50,56] and the structure changed from ELM to ELM (there was no data at the inner target). This same behavior has been observed in JT-60U also[55], as shown in Fig. 10. Here the data show the heat flux profile for three giant Type-1 ELMs in a neutral-beam heated $\mathrm{H}$ mode discharges, with the divertor geometry shown for reference. The apparently 
random variations in heat flux are evident, and it can be seen that the peak heat flux is displaced quite far from the separatrix. By sampling over a number of ELMs, Itami determined that these isolated peaks carried about $20-30 \%$ of the total power deposited on the plates by ELMs. Multiple peaks and radial structure in the divertor heat flux profile at the inner divertor target have also been observed in DIII-D[11]. In all these measurements, the rise in target plate surface temperature across the divertor is simultaneous to within the $100 \mu \mathrm{sec}$ temporal resolution of the cameras. One difference between the JT-60U data and the ASDEX-U data is the close proximity of the x-point to the target plates in JT-60U; the poloidal field is small and it is much easier to perturb the magnetic geometry in this case.

A different situation may occur in JET, where it is reported $[8,54]$ that the heat pulse at the inner target during an ELM can be shifted from the pre-ELM separatrix intercept by as much as $0.18 \mathrm{~m}$. In this case, the ELM appears to produce a shift in the location of the $x$-point due to the change in plasma position associated with the loss in stored energy during the ELM.

The occurrence of multiple peaks in the heat flux profile suggests the presence of islands or helical structures in the scrape off layer produced by the MHD instability. This in turn should result in toroidally asymmetric heat conduction to the target plates, though it would probably be masked by rapid toroidal rotation of the plasma. However, little data exists on toroidal asymmetries during ELMs. In DIII-D a toroidal array of net-current monitors is installed on the inner and outer divertor targets. Evidence for toroidal asymmetry in the net current during ELMs has been reported under some conditions with Type-1 ELMs[57]. Recently, the divertor heat flux during ELMs in DIII-D was measured simultaneously[18] using infrared cameras at two toroidal locations separated by $100^{\circ}$. There was considerable scatter in the heat flux ratio $\mathrm{R}=\Delta \mathrm{E}\left(60^{\circ}\right) / \Delta \mathrm{E}\left(165^{\circ}\right)$, but the peaking factor, defined as $\mathrm{R}_{\max } / \overline{\mathrm{R}}$, was less than 1.5:1 for the data set containing Type-1 ELMs. The authors attribute a large fraction of this peaking to noise in the measured heat flux.

Heat flux profiles have also been measured during Type-3 and during grassy Type-2 ELMs. In ASDEX-U with ICRF heating, it was observed that the heat pulse from Type-3 ELMs was peaked at the outer divertor target, in contrast with the observations for Type-1 ELMs. Since then, further measurements during neutral beam heated H-modes with Type-3 ELMs find that the heat pulse is too small to measure reliably, so no comparison can be made. In the DIII-D tokamak, it is observed that the heat pulse due to Type-3 ELMs is very similar in shape to that of 
Type-1 ELMs, but much smaller in amplitude[18]. Early heat flux measurements during grassy Type-2 ELMs in DIII-D found symmetric in/out pulses[51], but with the pulse at the inner target delayed by about $500 \mu \mathrm{sec}$ from that at the outer plate. Data from present machines with Type-1 ELMs show no evidence for in/out delays in the arrival of the heat pulse. The lack of time delay between pulses at each target plate for Type-1 ELMs has been used to support the claim that giant ELMs in JET begin at the $x$-point $[8]$.

During ELMs the particle flux to the divertor targets, divertor density, and divertor temperature increase as well. The rise in particle flux is beneficial for particle exhaust by divertor pumping[58]. In general, the particle flux does not increase as much as the heat flux (perhaps a factor of five to ten), but like the heat flux, the largest increase is at the inner target plate.

The particle flux during ELMs is routinely measured with Langmuir probes biased to collect ion saturation current. In JET, a set of triple-tip Langmuir probes has been used to measure the profiles of ion saturation current (ion flux) during Type-1 ELMs[59], as shown in Fig. 11. This plot was generated by radially sweeping the strike point across the probe tips; each ELM produces a spike in current, so the profile during the ELM is given by the envelope of the peak current. The ion flux profile in the quiescent periods between ELMs is given by the envelope of the minima, as shown. These data indicate that the ELMs broaden the particle flux profile at the outer target plates by about a factor of two.

It is much harder to measure the density and temperature at the divertor plates during an ELM with Langmuir probes because a very fast sweep of the I-V characteristic ( $t_{\text {sweep }}<0.1 \mathrm{msec}$ ) is required. On DIII-D a Thomson scattering system has been installed to measure the density and temperature in the divertor at eight positions along a vertical chord over a range of 0.01 to $0.20 \mathrm{~m}$ above the target plates. Careful positioning of the strike point relative to the Thomson chord allows measurement of the vertical profile of $\mathrm{n}_{e}$ and $\mathrm{T}_{\mathrm{e}}$ at $50 \mathrm{msec}$ intervals. In this way, the effect of single ELMs can be determined. Fig. 12 shows such a vertical profile along the outer divertor leg between (dashed) and during a typical Type-1 ELM. A large buildup in divertor density in front of the target plate is observed, while the change in electron temperature is not so large. These profiles resemble a very high density, high recycling attached divertor plasma where plasma pressure is nearly constant along field lines, but the value is much higher than in the quiescent phase, consistent with the higher energy flux (sheath heat flux is $\mathrm{P} \sqrt{\mathrm{T}}$ ). 
The large increase in particle flux at the divertor targets produces a significant increase in sputtering. With very large giant ELMs in JET, the impurity content in the main plasma is actually observed to increase after each event and the neutron reaction rate falls, presumably due to the large amount of sputtering[40]. Measurements in JT-60U show[60] that the carbon flux at the divertor targets during ELMs is proportional to the $\mathrm{H}_{\alpha}$ brightness and size of the ELM, but with apparently higher yield than during the quiescent phase, though effects due to changes in $T_{e}$ and ionizations/photon have not been included.

One interesting feature of ELMs reported in the literature[54, 59, 61] are so-called "negative" $\mathrm{H}_{\alpha}$ spikes, which are observed on certain viewing chords passing through the divertor leg during high density or detached plasma operation with deuterium gas puffing. These dips in emission result from the ELM heat pulse reattaching the plasma to the target plates. When detached, the ionization region moves off the divertor target and towards the $\mathrm{x}$-point region (a divertor or $\mathrm{x}$-point Marfe) due to radiative cooling of the divertor plasma. This can produce a rising $\mathrm{H}_{\alpha}$ signal on chords which pass through x-point region. When an ELM occurs, the heat pulse can reattach the divertor plasma, pushing the ionization region back near the plate; $\mathrm{H}_{\alpha}$ drops along one chord and rises along another. In DIII-D, the inner divertor leg is often detached, even without extra gas puffing[62], and the $\mathrm{H}_{\alpha}$ emission as viewed from a chord along this inner leg drops during the ELM pulse while other channels simultaneously rise. This spatial shift in the location of the radiating region can be seen with TV cameras viewing visible lines of carbon emission as well[63].

The in-out asymmetry of particle and heat flux profiles during Type-1 ELMs remains a mystery. Why is it reversed from the steady flux in between ELMs? No satisfactory explanation exists. Data from JT-60U show that it does not depend on the direction of the ion $\nabla \mathrm{B}$ drift[55], as do the steady fluxes between ELMs. If the ELM represents the sudden expulsion of plasma across the separatrix, then why doesn't the flux look like that for the steady H-mode or L-mode case, with more flow to the outer targets than the inner? Numerical simulations of ELMs in which the poloidally uniform cross-field particle and energy transport are suddenly increased everywhere in the SOL, find only a weak asymmetry, with slightly more heat and particles going to the inside target.

\section{Scaling with plasma parameters}


ELMs could pose a serious problem in future high power density fusion reactors, depending on the size of the heat pulse produced. In ITER, if an ELM produced a loss of $10 \%$ of the stored energy, then the power loading on the divertor surfaces would be high enough to damage the divertor targets. Figure 13 shows the expected lifetime of divertor target material in terms of the number of heat pulses vs. size of the heat pulse, assuming $3 \mathrm{~cm}$ thick carbon tile armor. With a total stored energy of about $1.2 \mathrm{GJ}$, a contact area of about $10 \mathrm{~m}^{2}$, a loss time of $1 \mathrm{msec}$, and assuming half the energy lost during the ELM is dissipated radiatively, then the realistic allowable size of ELMs in ITER is about $\triangle E / E \simeq 2.5 \%$. Most of the material would be lost via evaporation. In present tokamaks, this fractional energy loss per ELM lies someplace in between the losses expected for Type-1 and Type-3 ELMs. We should note that the important quantity here is the energy loss per ELM (not time averaged equivalent power unless it is comparable to the steady heating power - which it isn't) and the time over which the energy is lost (peak power). Also, since the curves show lifetime increasing nonlinearly as the ELM size drops, it becomes possible to trade ELM frequency and size even if $\triangle E / E \propto 1 / f$.

With these concerns, it becomes important to determine how the energy loss per ELM scales with heating power, stored energy, plasma current, or other design parameters. Only limited data exist on the scaling of ELM size, and very little comparative work between various machines has been completed. The ITER EDA has recently expanded its edge physics database to include ELM effects, but due to diagnostic limitations, most of the variables have to do with $\mathrm{H}_{\alpha}$ measurements (frequency, duration, pulse height, etc.); more work is clearly needed in this area.

Most of the existing scaling data are for Type-1 ELMs because the energy dump and divertor heat pulse are large enough to be easily measured. In DIII-D, initial studies[11, 64] of the scaling of ELM size with neutral beam heating power, $\mathrm{P}_{\mathrm{nbi}}$, found that the frequency, $f$, was proportional to $P_{n b i}$, as in Fig. 3 , while the energy loss per ELM as measured by a change in the stored energy, varied as $1 / \mathrm{P}_{\mathrm{nbi}}$. This gave a time-average energy loss per ELM which was nearly independent of beam power. Limited measurements of the divertor heat flux due to the ELMs also showed a steadily declining amplitude with increasing heating power.

More recently, studies of energy loss per Type-1 ELM were undertaken in ASDEX-U[29] and these data were compared with results from newer studies in DIII-D[18]. In this more recent work, better thermographic analysis of the divertor heat flux made possible direct comparison of the energy deposited on the target 
plates between the two machines. The results appear in Fig. 14. The ASDEX-U data show that the energy loss per ELM on the plates, about $7 \mathrm{~kJ}$ out of $380 \mathrm{kj}$ (at $4 \mathrm{MW}$ ) to $580 \mathrm{~kJ}$ (at $7.5 \mathrm{MW}$ ) total stored energy (a), is nearly constant with power, so that the fractional energy loss per ELM declines about 0.06\%/MW. Because the ELM frequency increases linearly with heating power, the time-average power ( $\mathrm{f} \Delta \mathrm{E}$ ) due to the ELMs (b) is a constant fraction of the beam power (15\% and $2 \%$ at the inner and outer targets respectively). Similar results are obtained in DIII-D, Fig. 14(c,d); the fractional energy loss per ELM drops very slightly with heating power, though it is somewhat larger than in ASDEX-U $(\sim 25 \mathrm{~kJ}$ out of a total stored energy of 0.9 to 1.4 MJ). Since a similar increase in ELM frequency with power is observed, the timeaverage target-plate power in DIII-D also increases with heating power, though at a much smaller rate than in ASDEX-U due to the large initial offset at $\mathrm{P}_{n b i}=2.5 \mathrm{MW}$.

Scaling studies for Type-3 ELMs have been carried out on the TCV tokamak and similar results regarding the correlation between ELM frequency and energy loss as with Type-1 ELMs are obtained[19]. For these small and rapid ELMs, the power and particle loss for individual ELMs was too small to measure directly, so the average $\Delta \mathrm{E} / \mathrm{E}$ and $\Delta \mathrm{N} / \mathrm{N}$ for a number of ELMs was determined from the global particle and energy balance equations. It was assumed that the ELMS did not change the underlying particle or energy confinement of the ELM-free plasma, $\tau_{\mathrm{E} 0}$, so that the time-average change in $\mathrm{E}$ or $\mathrm{N}$ followed the relation

$$
<\dot{W}_{\text {eff }}>/ W=P_{\text {in }} / W-\frac{1}{\tau_{\mathrm{E} 0}}+(<\Delta W>/ W) f_{\text {elm }} \text {. }
$$

Their results showing the steady decrease in fractional amplitude of the Type-3 ELMs with ELM frequency appear in Fig. 15. In these experiments the ELM frequency was varied by making small changes in plasma shape. Given the usual correlation between Type-3 ELM frequency and input power, one would infer that the amplitude of Type-3 ELMs grows as the heating power is increased above the $\mathrm{H}$ mode threshold power.

Recently, Osborne[18] has begun to relate Type-1 ELM amplitude scaling from ASDEX-U, JET, and DIII-D. His data in Fig. 16, show that the fractional energy loss per ELM decreases with power, and that results from these three machines can be correlated if the amplitude is plotted vs. the normalized power $\left(\mathrm{P}_{\text {sep }} / \mathrm{A}_{\mathrm{sep}}\right)$ over the $\mathrm{H}$-mode power threshold. That is, $\Delta \mathrm{E} / \mathrm{E}$ decreases as $\mathrm{P}_{\text {sep }} /\left(\mathrm{A}_{\text {sep }} \times \mathrm{P}_{\mathrm{H}}\right)$ increases. Here he has not included the density variation, but rather assumed it affects the $\mathrm{H}$-mode threshold through the radiation loss from the core, which is used to obtain $\mathrm{P}_{\text {sep }}=$ $\mathrm{P}_{\mathrm{nbi}}-\mathrm{P}_{\mathrm{rad}}$. His reason for normalizing to the H-mode threshold power is the 
assumption that the bulk of the power loss comes from a period of L-mode confinement following the fast MHD event and the length of this period is set by the H-mode power threshold. His approach puts the ASDEX-U and JET data in the middle of the DIII-D data even though the size of the machines is very different. Carrying out this same analysis for the expected ITER parameters yields an expected ELM amplitude of $\triangle E / E=0.03$, which is just above the acceptable level, meaning that some way must be found to increase the ELM frequency and reduce the amplitude for ITER.

\section{ELM Control Experiments}

While understanding the scaling of ELM size with heating power and machine size is a necessary step for accessing the impact of ELMs on future larger machines, it would be better if active control of the ELMs could be achieved. ELM control experiments have been carried out in several tokamaks: DIII-D[6, 65], JFT-2M[66], COMPASS-D[67], indirectly in ASDEX-U[20, 68] and TCV[19]. These experiments have attempted to control the ELM behavior by changing the power flow through the edge plasma (DIII-D, ASDEX-U, and COMPASS-D), the plasma shape (DIII-D, TCV) or the magnetic structure or H-mode power threshold (JFT-2M, COMPASS-D). The results show that it is possible to change the frequency and amplitude of Type-3 ELMs, move the plasma from ELM-free to ELMy H-mode, switch from Type- 1 to grassy Type-2 ELMs, and switch from Type-1 to Type-3 ELMs, which gives some optimism that ELM control in future high power tokamaks such as ITER will be possible, though perhaps not easy.

One of the most straightforward ways to control ELM behavior is to vary the power flow through the edge plasma. This has been done very effectively on ASDEX-U, where a combination of deuterium and neon injection, along with divertor pumping was used to increase the radiative losses from the edge plasma to the point $\left(\mathrm{P}_{\mathrm{rad}}=70 \%\right)$ where the plasma was operating near $\mathrm{H}$-mode power threshold and Type-3 ELMs were produced. Fig. 17 from Kallenbach, et al. [68] shows how the ELM behavior and confinement varied as the power flow across the separatrix was reduced from well above to below the H-mode threshold level. The Type-3 ELMs were so small and so rapid in the $\mathrm{CDH}$ mode discharge (Completely Detached $\mathrm{H}$-mode) that no measurable heat pulse is observed on the divertor targets and the carbon sputtering is reduced significantly. In these experiments, the 
deuterium gas was injected near the midplane and feedback control was used to maintain a constant divertor neutral flux. The edge radiation was produced by neon injection near the midplane; feedback control was used to maintain a constant radiation loss.

Controlling the ELM behavior by controlling the power flow through the edge has good points and bad points. On the good side, lowering the power flow into the SOL helps the divertor problem, and doing so by impurity gas puffing is fairly robust and doesn't require complicated hardware. On the down side, operating with high radiating fractions may make the plasma more sensitive to disruptions, and producing adequate edge impurity radiation to bring the power down may require a higher impurity concentration than desired in terms of core fuel dilution. Also, the operating window for maintaining acceptable ELM characteristic may be rather narrow and hard to maintain. However, the results on ASDEX-U look encouraging.

Other, more subtle means of ELM control consist of the application of perturbing magnetic fields to the edge plasma. In COMPASS-D, external coils were used to introduce a broad spectrum of relatively low- $m(m<7)$ perturbations [67] and this increased the frequency and reduced the amplitude of Type-3 ELMs. The reason for the change is not clear, but speculation centers on the perturbation either increasing the $\mathrm{H}$-mode power threshold by increasing radial transport in the edge (recall that Type-3 ELM frequency decreases as $\mathrm{P}_{\text {sep }}-\mathrm{P}_{\mathrm{H}}$ increases) or the perturbation directly affecting the MHD stability of the underlying ELM modes. In JFT-2M, use of an ergodic magnetic limiter (EML) to introduce high $m, n$ perturbations to the plasma edge was also observed to increase the ELM frequency[66]. This perturbation also expanded the operating region for obtaining ELMy $\mathrm{H}$-mode discharges, as shown in Fig. 18; with increasing EML current, Type-3 ELMy H-mode operation could be maintained with up to $50 \%$ more heating power. In this case, the H-mode power threshold was also increased somewhat. Thus, it may be that the effect on the ELMs resulted from a decrease in $P_{\text {sep }}-P_{H}$ rather than a fundamental change in the ELM stability. In this regard, the COMPASS-D and JFT-2M results may be very similar.

ELM control via ECH heating of the edge plasma has also been demonstrated in DIII-D[69] and COMPASS-D[70] with the results pretty much in agreement with what would be expected for the application of significant heating power to the edge plasma. In COMPASS-D, $200 \mathrm{~kW}$ of 2nd harmonic ECH was applied to an ohmic Hmode discharge $\left(I_{p}=200 \mathrm{kA}\right)$ and the frequency of Type-3 ELMs decreased, as shown in Fig. 19. In the DIII-D experiments, application of ECH just inside the separatrix 
increased the frequency of Type-1 ELMs, probably due to increasing the total power flowing through the edge plasma ( $\left.\mathrm{P}_{\mathrm{ech}}=1 \mathrm{MW}, \mathrm{P}_{\mathrm{nbi}}=5 \mathrm{MW}\right)$. One surprise in this experiment was that heating the plasma just outside the separatrix decreased the ELM frequency by nearly a factor of two, though it was suggested that the external heating lowered the edge pressure gradient by increasing $T_{e}$ on the separatrix. However, no measurements of the edge temperature gradients were obtained.

Plasma shaping offers another possibility for ELM control, but only within the limits of the basic machine design. With Type- 1 giant ELMs, changing the plasma elongation or triangularity can affect the ballooning mode stability through the edge magnetic shear, and this can directly affect the ELM frequency and amplitude. In DIII-D increasing the triangularity has been used to suppress Type-1 ELMs and move to an operating regime with very small Type-2 grassy ELMs only[6]. Alternatively, changing the $x$-point configuration from one in which the H-mode power threshold is low (ion $\nabla \mathrm{B}$ drift toward the null) to one in which it is considerably higher ( ion $\nabla B$ drift away from the $x$-point or a double-null divertor) can delay the onset of Type-1 ELMs or bring on the onset of Type-3 ELMs, depending on how close to the $\mathrm{H}$-mode threshold one is operating. In $\mathrm{TCV}$, very small shifts in the magnetic separatrix have been used to alternately stabilize or destabilize Type3 ELMs and thereby achieve density control in ohmic H-mode discharges[19]. With the separatrix shifted by about $1 \mathrm{~cm}$ or less to put the active $x$-point in the favorable direction, Type-3 ELMs were suppressed (increased $\mathrm{P}-\mathrm{P}_{\mathrm{H}}$ ) and the density rose, while

placing the active $\mathrm{x}$-point in the unfavorable direction (decreased $\mathrm{P}-\mathrm{P}_{\mathrm{H}}$ ) produced rapid ELMs which lowered the plasma density. However, it was not known how this small change in plasma magnetic equilibrium changed the power and particle flow to the divertor.

\section{Discussion and Summary}

ELMs have been the focus of numerous studies since the discovery of the $\mathrm{H}$ mode, but interest in them has grown since the start of the ITER project, when it became clear that repetitive large ELMs dumping $10 \%$ of the stored energy would present problems for divertor lifetime. Three common types have been identified and some of the fundamental properties of each have been identified. Type-1 giant ELMs are observed at power levels well above (1.5× or more) the $\mathrm{H}$-mode power threshold and their repetition rate increases with power. Type-2 grassy ELMs are 
observed in highly shaped plasmas whose configuration allows access to the 2nd stable ballooning region. Type-3 ELMs occur just above the H-mode power threshold $\left(\mathrm{P} / \mathrm{P}_{\mathrm{H}} \leq 1.2\right.$ based on ASDEX- $\mathrm{U}$ data) and their frequency decreases with increasing heating power. Type-2 ELMs produce the smallest loss of stored energy $(\Delta \mathrm{W} / \mathrm{W}<<0.01)$, with Type-3 producing somewhat larger loss $(\Delta \mathrm{W} / \mathrm{W}<0.02)$ and Type-1 the largest at ( $\Delta W / W \sim 0.01-0.1$ or more).

The size and shape of the divertor heat pulse during ELMs has been measured on several divertor tokamaks and it is found that most of the power is deposited on the inner target plates, which is just the opposite of what is observed for L-mode plasmas or in the quiescent times between ELMs in H-mode discharges. No satisfactory explanation exists for this behavior, which seems to suggest that the divertor heat pulse is not due simply to a brief return to L-mode confinement. Indeed, some of the heat flux profiles recorded in ASDEX[50], DIII-D[11], and JT60U[55] show radial structure which is strongly suggestive of helical structures or other large scale MHD distortion of the magnetic topology.

Scaling studies of the size of the divertor heat pulse on ASDEX-U and DIII-D show favorable projections for future high power machines: the size of the ELM pulse in a given machine does not grow with heating power, but rather the frequency of the ELMs increases. Since divertor plate damage by ELMs is largely governed by the peak energy density deposited by each ELM rather than the timeaverage power, this is a good result. However, we still cannot reliably predict the absolute size or frequency of ELMs expected in a totally new device, such as the proposed ITER tokamak. This is due in part to our poor understanding of all the factors which govern ELM behavior (recycling, plasma shape, divertor geometry), and in large part to uncertainties in predicting accurately the $\mathrm{H}$-mode power threshold.

Our inability to predict the size of ELMs in future machines places importance on figuring out ways to control the type of ELMs and their amplitude and frequency. Most of the know techniques involve controlling either the $\mathrm{H}$-mode power threshold or the power crossing the separatrix; neither quantity may be a free parameter in an operating fusion reactor. One of the more promising results on ELM control so far is the CDH mode developed in ASDEX-U, in which edge impurity radiation lowers the power crossing the separatrix to the point where small Type-3 ELMs can be obtained[20]. However, the operating window for this regime appears quite narrow and its compatibility with high power operation is uncertain. 
Rapid progress on ELM scaling and ELM control awaits a better theoretical understanding of the nonlinear dynamics of the ELM. Present intuition regarding ELM behavior is based largely on linear stability calculations, which only give information on the start of the collapse. Developing a model that explains the depth of the collapse of the edge plasma as well as the onset condition is the key to being able to predict and control the size of ELMs in future machines. Along these lines, experimental studies of ELMs should place more emphasis on how the plasma parameters just inside the separatrix evolve after the start of the ELM, rather than focusing largely on precursors or other aspects governing only the onset conditions. As edge diagnostic improve, we may be able to make measurements which the theory community can use to produce a better nonlinear model of the ELM.

\section{Acknowledgments}

The author wishes to acknowledge support from several individuals who contributed data and interpretation of the data for this paper. They are: Nobu Asakura, Hans-Stephan Bosch, Albrecht Herrmann, Kiyoshi Itami, Arne Kallenbach, Michael Kaufmann, Charlie Lasnier, Tony Leonard, Hans Lingertat, Guy Matthews, Raymond Monk, William Morris, Tom Osborne, Horst Pacher, Richard Pitts, Wolfgang Suttrop, and Hartmut Zohm. This work was performed under the auspices of the US Department of Energy by LLNL under contract

W-7405-ENG-48. 



\section{References}

1. F. Wagner, G. Becker, K. Behringer et al., Phys. Rev. Lett. 49, 1408-1412 (1982).

2. S. M. Kaye, M. G. Bell, K. Bol et al., J. Nucl. Mater 121, 115-125 (1984).

3. N. Ohyabu, K. H. Burrell, J. DeBoo et al., Nucl. Fusion 25, 49-63 (1985).

4. C. E. Bush, R. J. Goldston, S. D. Scott et al., Physical Review Letters 65, 424-427 (1990).

5. E. J. Doyle, R. J. Groebner, K. H. Burrell et al., Phys. Fluids B 3, 23002307 (1991).

6. T. Ozeki, M. S. Chu, L. L. Lao et al., Nucl. Fusion 30, 1430 (1990).

7. V. V. Parail, B. Balet, P. Bak et al., in 22nd EPS Conf. on Cont. Fusion and Plasma Phys., Bournemouth, 1995), p. I-013.

8. J. Lingertat, B. Alper, S. Ali-Arshad et al., in 22nd EPS Conf. on Cont. Fusion and Plasma Phys., Bournemouth, 1995), p. III-281.

9. R. Moyer, et al., in These proceedings, St. Raphael, 1996).

10. M. A. Mahdavi, D. Hill, S. L. Allen et al., J. Nucl. Mater. 162-164, 245-250 (1989).

11. D. N. Hill, A. Futch, A. W. Leonard et al., J. Nucl. Mater 196-198, 204-209 (1992).

12. H. Zohm, T. H. Osborne, K. H. Burrell et al., Nucl. Fusion 35, 543-550 (1995).

13. D. N. Hill, T. W. Petrie, J. N. Brooks et al., in Proc. 13th Int. Conf. on Plasma Physics and Controlled Nuclear Fusion Research, Washington, D.C., 1990), Vol. 3, p. 487-501.

14. A. Herrmann, W. Junker, K. Gunther et al., in 20th EPS Conf. on Cont. Fusion and Plasma Phys., Lisbon, 1993), p. II-567.

15. H. D. Pacher, I. JCT, H. Teams, , San Diego, 1995).

16. M. Kaufmann, H. S. Bosch, A. Field et al., Plasma Phys. Control. Fusion 35, 205214 (1993).

17. F. Ryter, K. Büchl, C. Fuchs et al., Plasma Phys. Control. Fusion 36, 99-104 (1994).

18. A. W. Leonard, W. Suttrop, A. Herrmann et al., in 12th Int. Conf. on Plasma Surface Interactions, 1996).

19. H. Weisen, M. J. Dutch, A. Pochelon et al., in 22nd EPS Conf. on Cont. Fusion and Plasma Phys., Bournemouth, 1995), p. III-113.

20. O. Gruber, A. Kallenbach, M. Kaufmann et al., Phys. Rev Lett. 74, 4217-4220 (1995).

21. A. Kallenbach, R. Dux, V. Mertens et al., Nucl. Fusion 35, 1231-1246 (1995).

22. H. Zohm, Plasma Phys. Control. Fusion 38, 105-128 (1996).

23. ASDEX Team, Nuclear Fusion 29, 1959-2040 (1989).

24. A. Tanga, for the JET Team, Plasma Phys. Control. Fusion 36, 39-53 (1994). 
25. H-mode Database Working Group, in 20th European Conf. on Plasma Physics., Lisbon, 1993), Vol. 17C Pt. 1, p. 103.

26. R. Mueller, G. Janeschitz, P. Smeulders et al., Nucl. Fusion 27, 1817 (1987).

27. D. A. Content, H. W. Moos, M. E. Perry et al., Nucl. Fusion 30, $701-715$ (1990).

28. M. G. O'Mullane, H. Chen, I. H. Coffey et al., in 22nd EPS Conf. on Cont. Fusion and Plasma Phys., Bournemouth, 1995), p. III-121.

29. W. Suttrop, K. Schonmann, J. Schweinzer et al., in 22nd EPS Conf. on Cont. Fusion and Plasma Phys., Bournemouth, 1995), p. III-237.

30. A. L. Colton, S. Ali-Arshad, P. Cripwell et al., J. Nucl. Mater. 196-198, 404-408 (1992).

31. A. L. Colton, L. Porte, in 20th EPS Conf. on Cont. Fusion and Plasma Phys., Lisbon, 1993), p. I-11.

32. K. H. Burrell, T. N. Carlstrom, E. J. Doyle et al., Phys. Fluids B 2, 1405-1410 (1990).

33. N. C. Hawkes, D. B. Bartless, D. J. Campbell et al., in 22nd EPS Conf. on Cont. Fusion and Plasma Phys., Bournemouth, 1995), p. III-261.

34. M. Valovic, M. Edwards, D. Gates et al., in 21st EPS Conf. on Cont. Fusion and Plasma Phys., Montpellier, 1994), p. 318-321.

35. DIII-D Team, in Proc. 13th INt. Conf. on Plasma Physics and Controlled Nuclear Fusion Research, Washington, D.C., 1990), Vol. 1, p. 69-91.

36. O. Pogutse, J. G. Cordey, W. Kerner et al., in 22nd EPS Conf. on Cont. Fusion and Plasma Phys., Bournemouth, 1995).

37. P. Gohil, M. A. Mahdavi, L. Lao et al., Phys. Rev. Letts. 61, 1603-1606 (1988).

38. H. Zohm, K. Buchl, A. R. Field et al., in 20th EPS Conf. on Cont. Fusion and Plasma Phys., Lisbon, 1993), p. I-19.

39. T. H. Osborne, Private Communications, 1996.

40. T. C. Hender, B. Alper, N. Deliyanakis et al., in 22nd EPS Conf. on Cont. Fusion and Plasma Phys., Bournemouth, 1995), p. I-029.

41. H. Zohm, F. Ryter, C. Fuchs et al., Plasma Phys. Control. Fusion 36, 129-134 (1994).

42. O. Vollmer, R. Aratari, F. Ryter et al., in 17th EPS Conf. on Controlled Fusion and Plasma Physics, Amsterdam, 1990), Vol. I, p. 295.

43. K. McCormick, M. Besserodt-Weberpals, E. R. Müller et al., Journal of Nuclear Materials 176\&177, 89-101 (1990).

44. M. Kikuchi, M. Sato, Y. Koide et al., in 20th EPS Conference on Controlled Fusion and Plasma Physics, Lisboa, 1993), p. I-179.

45. M. E. Rensink, S. L. Allen, A. H. Futch et al., Physics Fluids. B 5, 2165-2175 (1993). 
46. R. J. Buttery, D. A. Gates, A. Colton et al, in 22nd EPS Conf. on Cont. Fusion and Plasma Phys., Bournemouth, 1995), p. III-273.

47. D. P. Coster, B. Braams, J. Neuhauser et al., in 1994 EPS, 1994).

48. J. M. Noterdaeme, M. Brambilla, D. Coster et al., in 21st EPS Conf. on Cont. Fusion and Plasma Phys., Montpellier, 1994), p. 842-845.

49. D. P. Coster, R. Schneider, J. Neuhauser et al., in Plasma Edge Theory Conference, Asilomar, 1995).

50. E. R. Mueller, M. Keilhacker, K. Steinmetz, J. Nucl. Mater. 121, 138-143 (1984).

51. D. N. Hill, T. Petrie, M. A. Mahdavi et al., Nucl. Fusion 28, 902-907 (1988).

52. A. Herrmann, M. Laux, D. Coster et al., J. Nucl. Mater. 220-222, 543-547 (1995).

53. K. Itami, M. Shimada, N. Hosogane, J. Nucl. Mater 196-198, 755-758 (1992).

54. J. Lingertat, J. Nucl. Mater (1996).

55. K. Itami, N. Hosagone, N. Asakura et al., J. Nucl. Mater. 220-222, 203-207 (1995).

56. G. Fussmann, U. Ditte, W. Eckstein et al., J. Nucl. Mater. 128 \&129, 350-358 (1984).

57. T. E. Evans, C. J. Lasnier, D. N. Hill et al., J. Nucl. Mater. 220-222, 235-239 (1995).

58. C. C. Klepper, J. T. Hogan, P. K. Mioduszewski et al., J. Nucl. Mater. 196-198, 10901095 (1992).

59. R. Monk, , 1996).

60. S. Sakurai, N. Asakura, .

61. T. W. Petrie, J. Nucl. Mater. (1992).

62. R. Maingi, J. Nucl. Mater (1994).

63. V. Mertens, H. S. bosch, K. Buchel et al., in 21st EPS Conf. on Cont. Fusion and Plasma Phys., Montpellier, 1994), p. 326-329.

64. R. Stambaugh, (old).

65. J. Lohr, et al., , 1991).

66. T. Shoji, H. Tamai, Y. Miura et al., J. Nucl. Mater. 196-198, 296-300 (1992).

67. T. N. Todd, C. Team, Plasma Phys. Control. Fusion 35, 231-240 (1993).

68. A. Kallenbach, R. Dux, V. Mertens et al., Nucl. Fusion 35, 1231-1246 (1995).

69. T. C. Luce, R. A. James, A. Fyaretdinov et al., in 13th International Conf. on Plasma Physics and Cont. Nucl. Fusion Research (IAEA, Washington, DC, 1990), Vol. 1, p. 631.

70. A. W. Morris, A. M. Edwards, T. C. Hender et al., in IAEA Conf. on Controlled Fusion Research (IAEA, Seville, Spain, 1994), p. 365-374. 
Tables

Table 1: ELM Activity reported for presently operating divertor tokamaks

\begin{tabular}{|l|l|l|l|}
\hline Device/Heating & Ohmic Heating & RF Heating & Neutral beams \\
\hline ASDEX-Upgrade & & Type-1, 3, dithering & Type-1 \& 3, dithering \\
\hline Alcator C-mod & Type-3 & Type-1, Type-3 & \\
\hline COMPASS-D & Type-1,3 & & \\
\hline DIII-D & dithering & & Type-1,2,3, dithering \\
\hline JET & & Type-1,3 & Type-1,3 \\
\hline JFT-2m & & & Type-1 \& 3 \\
\hline JT-60U & & Type-1 & Type-1, 2, \& 3 \\
\hline TCV & Type-2,3, dithering & & \\
\hline
\end{tabular}




\section{Figure Captions}

Fig. 1. An H-mode discharge in JET showing Giant Type-1 ELMs.

Fig. 2 Radial profiles of electron density and temperature before $(2510 \mathrm{msec})$ and during a Type-1 ELM in DIII-D starting at $2559.9 \mathrm{msec}$.

Fig. 3 Dependence of Type-1 ELM frequency on plasma current and beam power in (a)DIII-D, and (b), in JET.

Fig. 4. An H-mode discharge in DIII-D showing Type-3 ELMs with frequency decreasing as the beam power increases.

Fig. 5. $\mathrm{H}_{\alpha}$ traces from several tokamaks showing other types of ELM activity or fluctuations similar to ELMs. (a) Type-2 ELMs in DIII-D, (b) several types of ELMs in TCV, (c) giant ELMs in JET, and (d) L-H-L dithering in ASDEX-U.

Fig. 6. Power scan during an ASDEX-U H-mode discharge showing transition from Type-3 ELMs to Type-1 with steadily increasing frequency.

Fig. 7 Operating space for obtaining $\mathrm{H}$-mode and different kinds of ELMs in ASDEX-U. Space between shaded regions is ELM-free and Type-3 ELM region. 0 -ohmic, $\Delta$ - L-mode, $\bullet$ - ohmic H-mode, - ELM-free , + dither, $\times$ Type-3, $\mathbf{\square}-\mathrm{ICRH}, \Delta$ Type-1

Fig. 8 Midplane Thomson scattering data in DIII-D showing a broader density SOL after the onset of rapid ELMs at $3 \mathrm{sec}$. The electron temperature in the SOL is largely unaffected in the time-average sense. Separatrix is at $80 \mathrm{~cm}$.

Fig. 9 Divertor heat flux profiles during ELMs in AUG as measured by an infrared line array detector. Top: 3-d isometric plot showing temporal evolution, bottom: radial profiles at selected times showing fall-off lengths in mm.

Fig. 10 Bifurcated divertor heat flux during ELMs in JT-60U. Top-divertor magnetic flux surfaces showing location of x-point and separatrix. Bottom, profiles as measured with IR TV camera for three different ELMs.

Fig. 11 Radial profile of ion saturation current in the JET divertor during a radial sweep showing two characteristic fall-off lengths for the ELM peaks and the quiescent $\mathrm{H}$-mode times between ELMs.

Fig. 12 Vertical profiles of density and temperature along the outer divertor leg in DIII-D as measured by Thomson scattering. Dashed-quiescent H-mode between ELMs, solid-during a Type-1 ELM.

Fig. 13 Graph showing carbon PFC lifetime vs. size of heat pulse in ITER. From H. Pacher. 
Fig. 14. Power scaling of energy loss per ELM and time-averaged power due to Type-1 ELMs in ASDEX-U and DIII-D.

Fig. 15 Variation of Type-3 ELM particle (a) and energy (b) loss with ELM frequency.

Fig. 16 Initial database of ELM size scaling with power normalized to the $\mathrm{H}$-mode power threshold. Point for ITER determined assuming published $\mathrm{H}$-mode threshold power scaling.

Fig. 17 ELM control in AUG via neon puffing to reduce the power flow through the edge plasma. Type-3 ELMs are obtained when the power crossing the separatrix is near the $\mathrm{H}$-mode threshold, $\mathrm{P}_{\mathrm{thr}}^{\mathrm{H}-\mathrm{L}}$.

Fig. 18 ELM control in JFT-2M: Increasing ergodic magnetic limiter current increases the power range over which ELMy H-modes can be obtained.

Fig. 19 ELM control in COMPASS-D: ELM frequency decreases with the application of $200 \mathrm{~kW}$ of $\mathrm{ECH}$ power. 


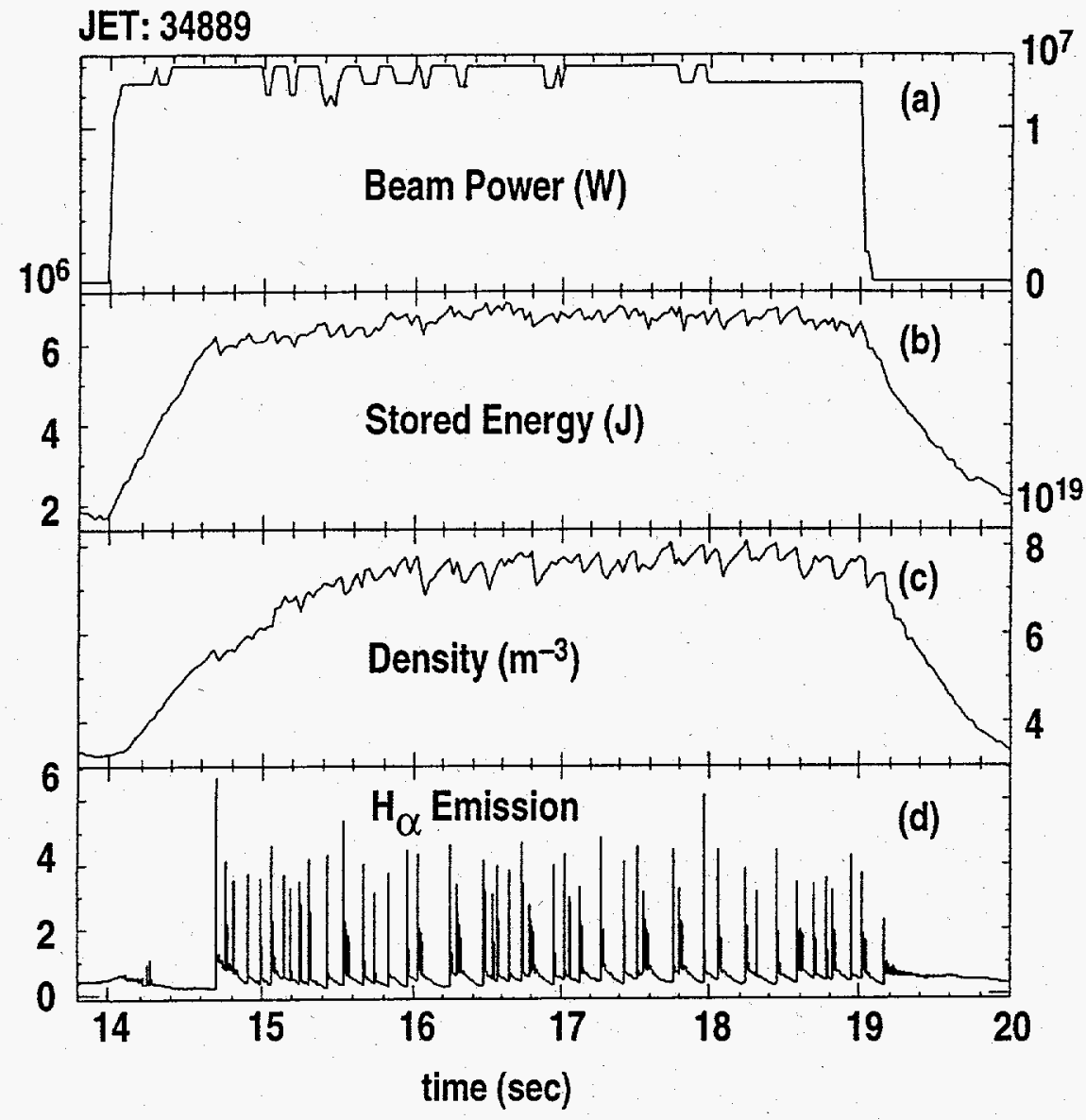

Fig. 1 

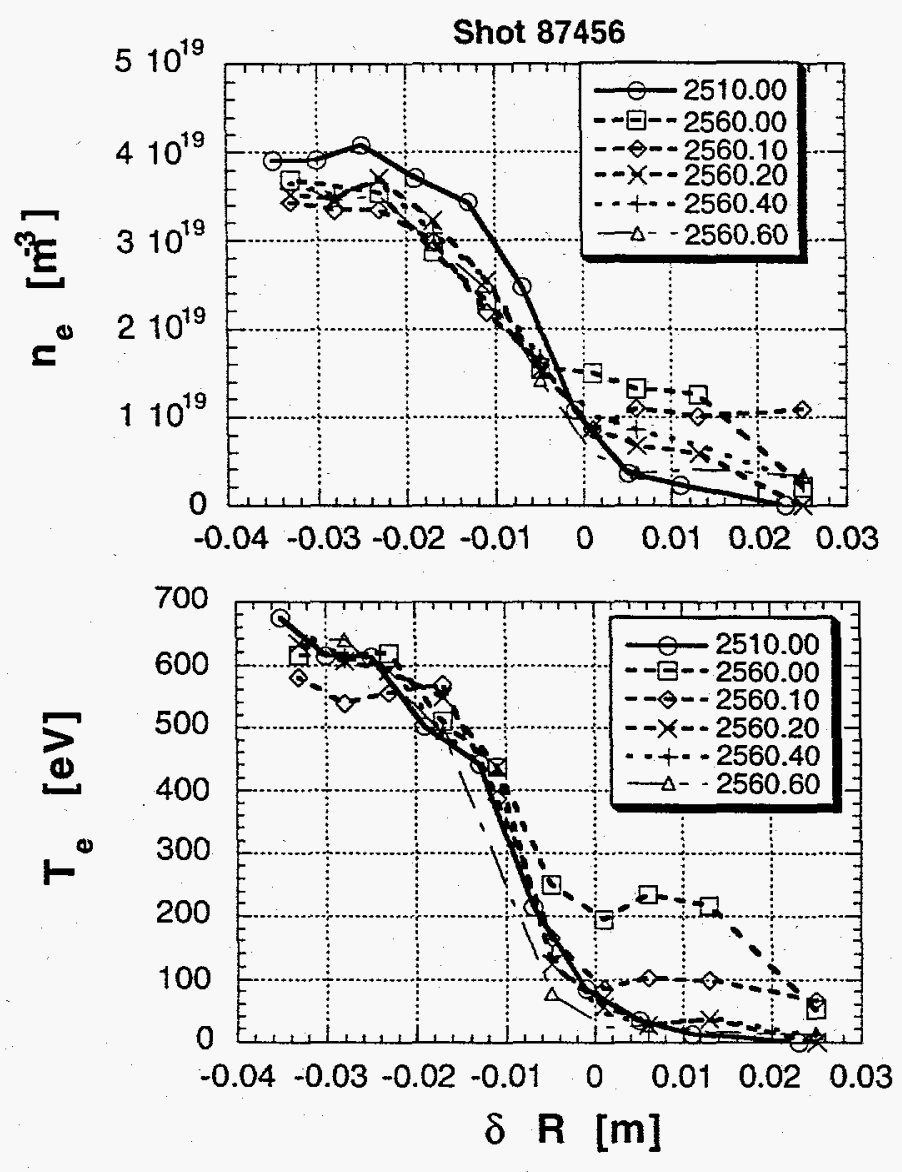

Fig. 2 

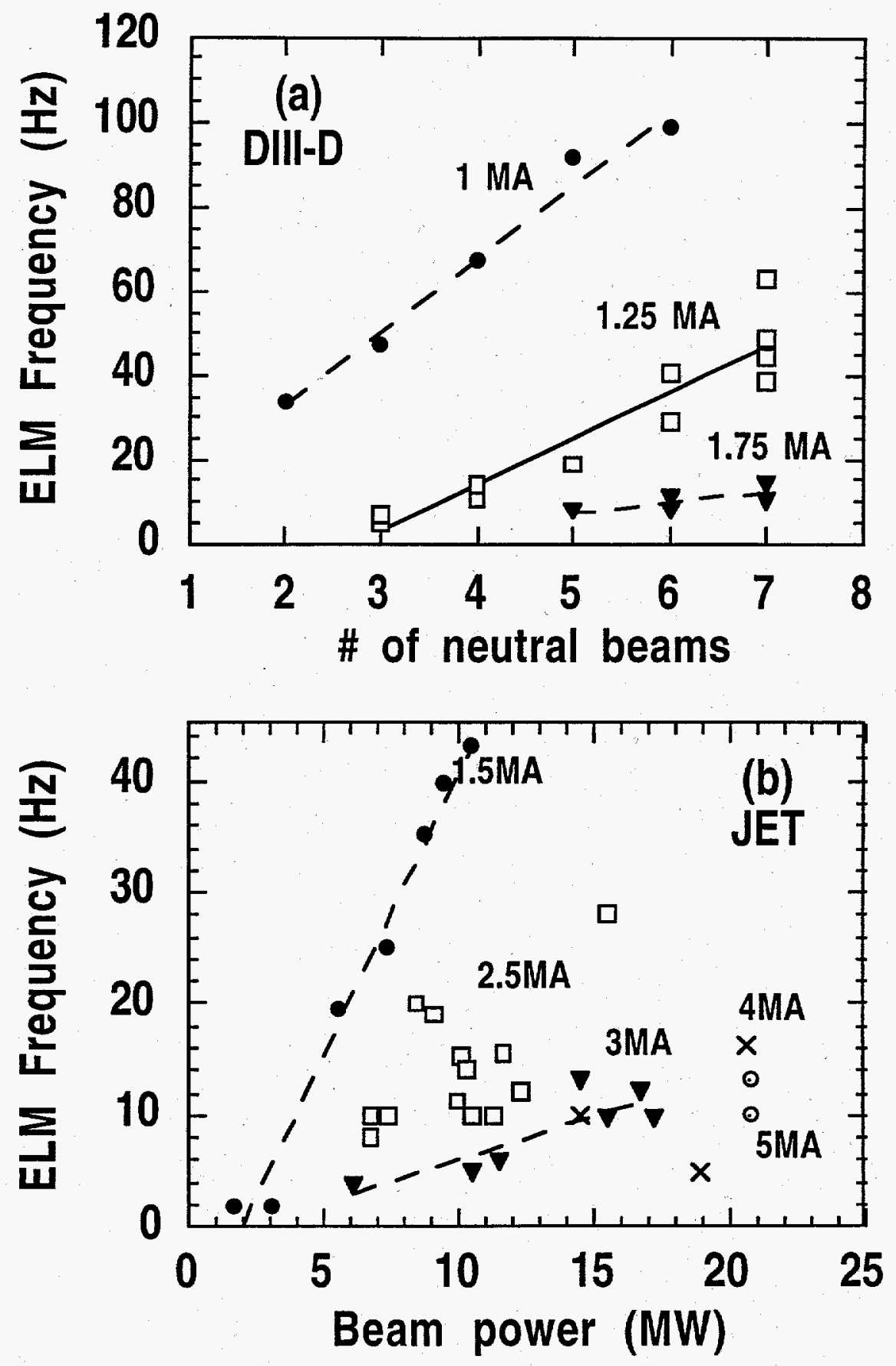

Fig. 3 


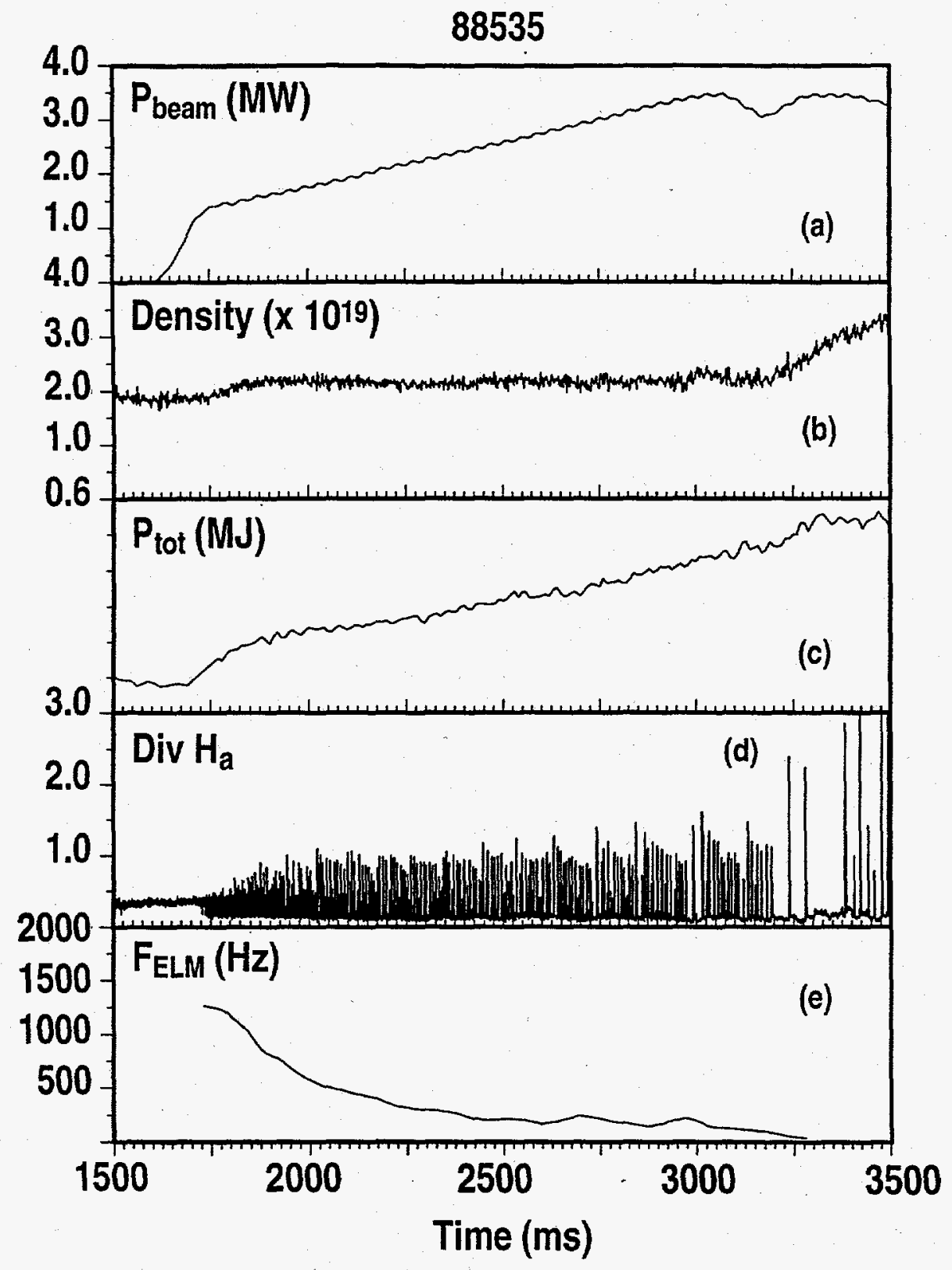

Fig. 4 

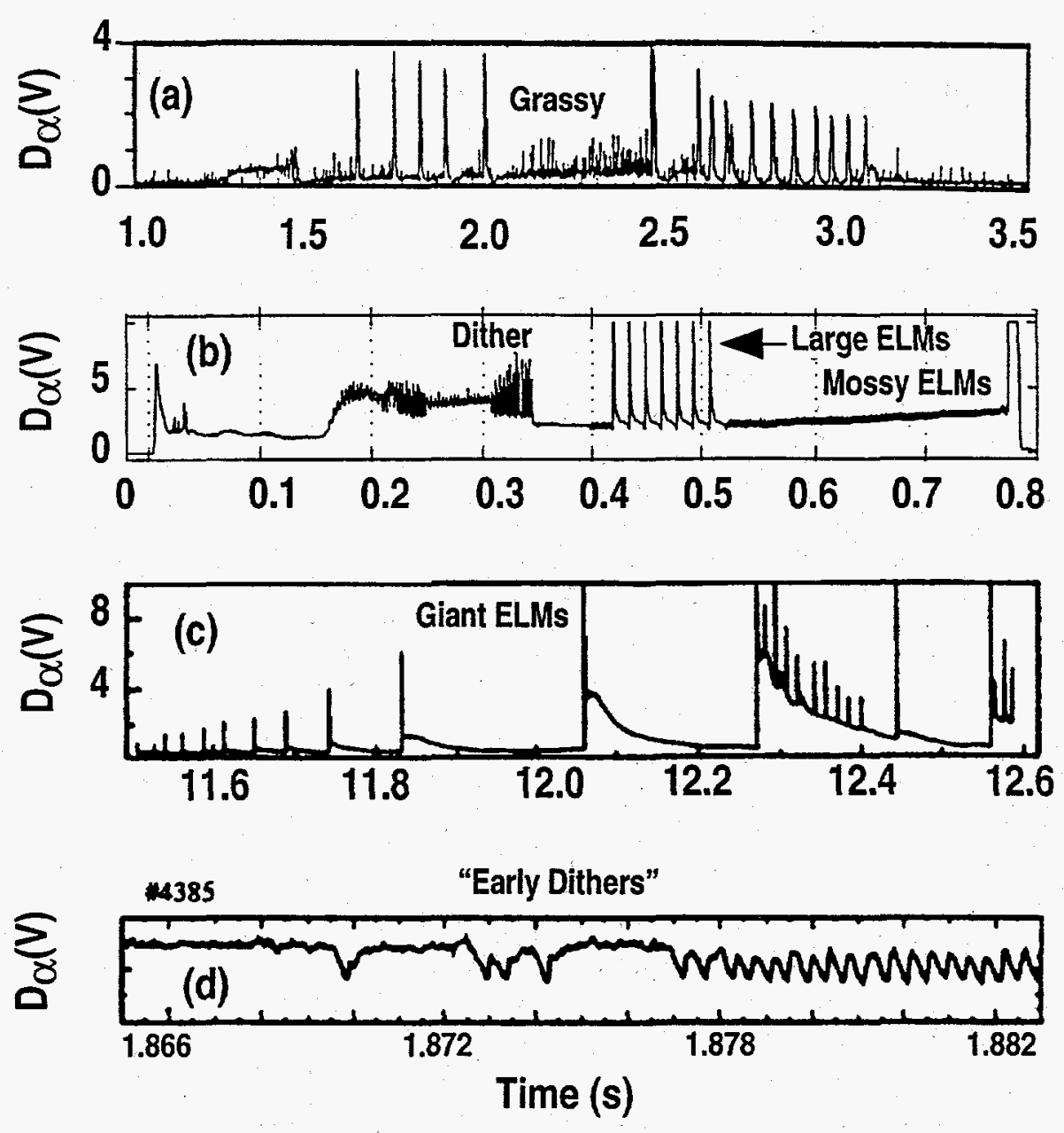

Fig. 5 


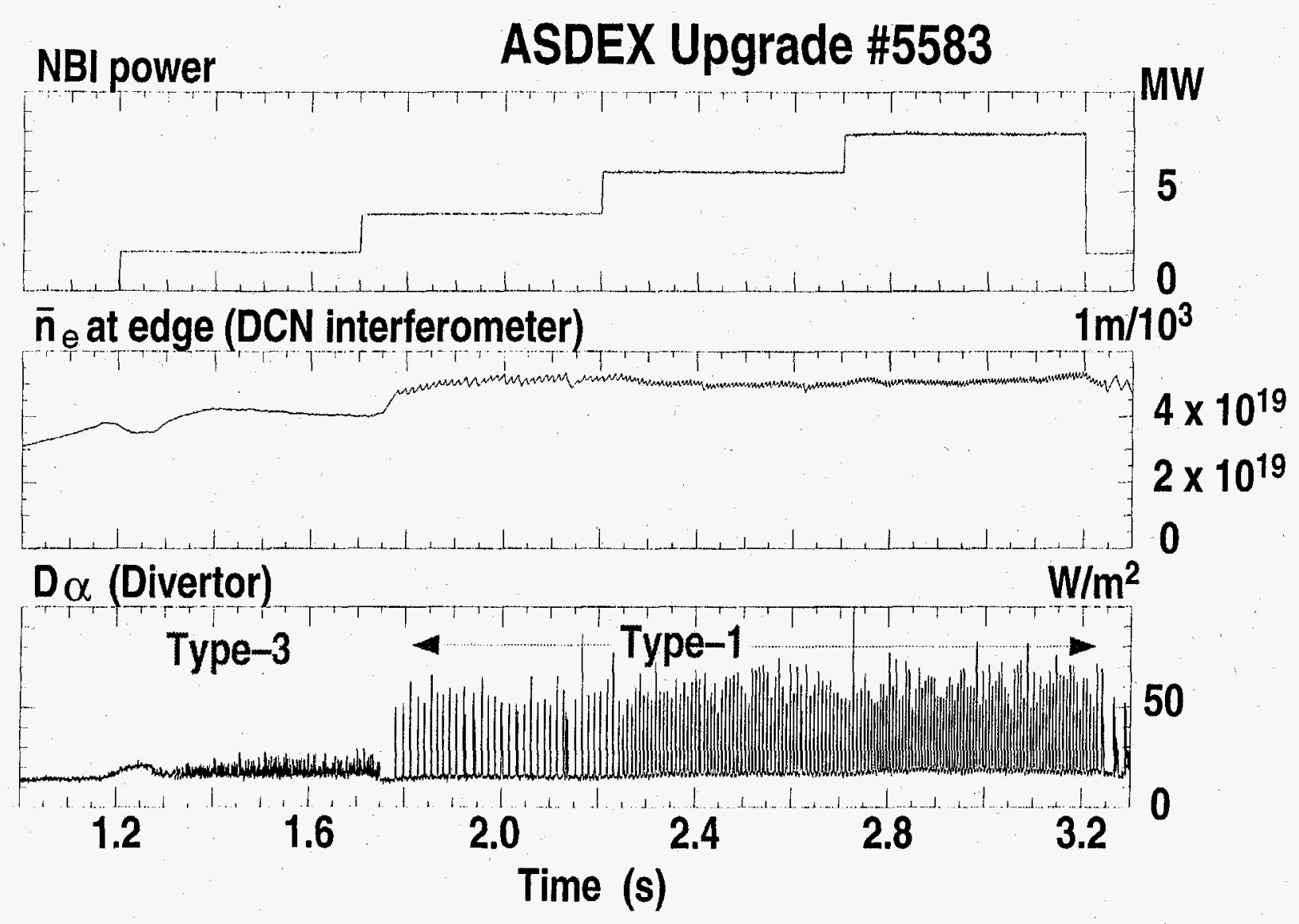

Fig. 6 


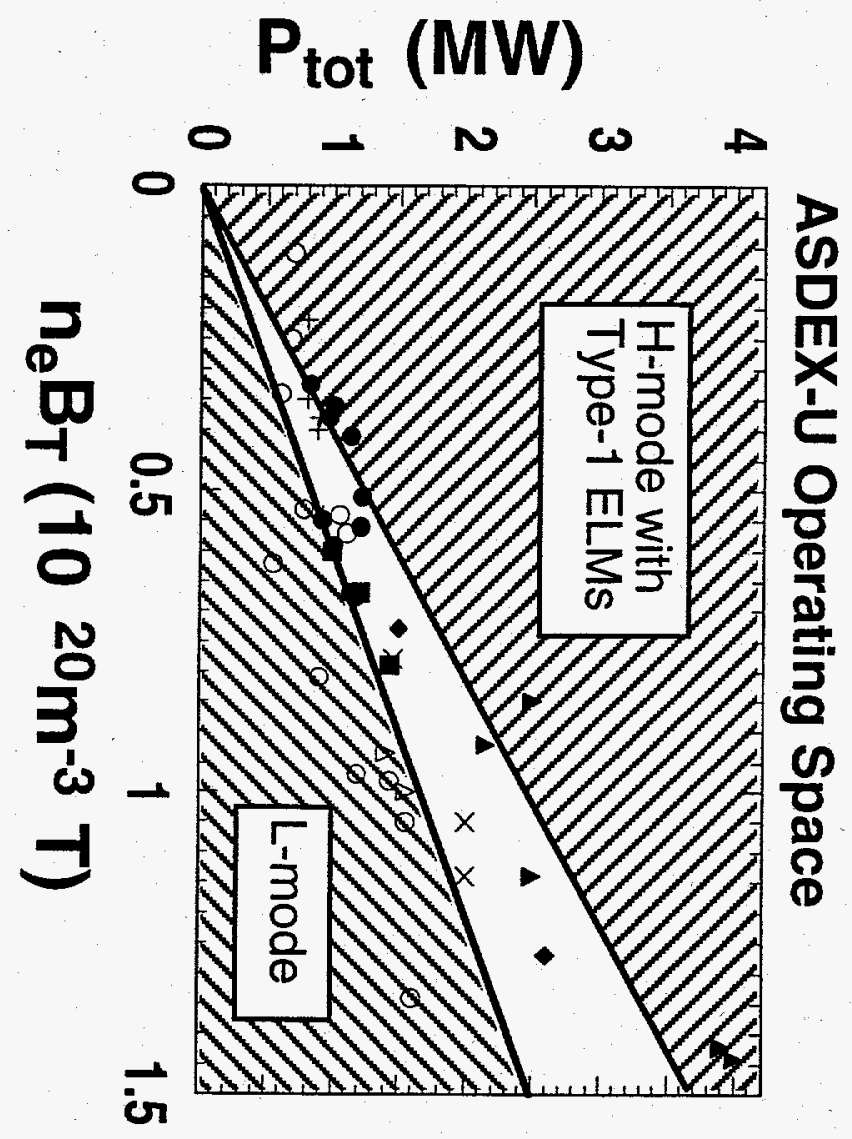




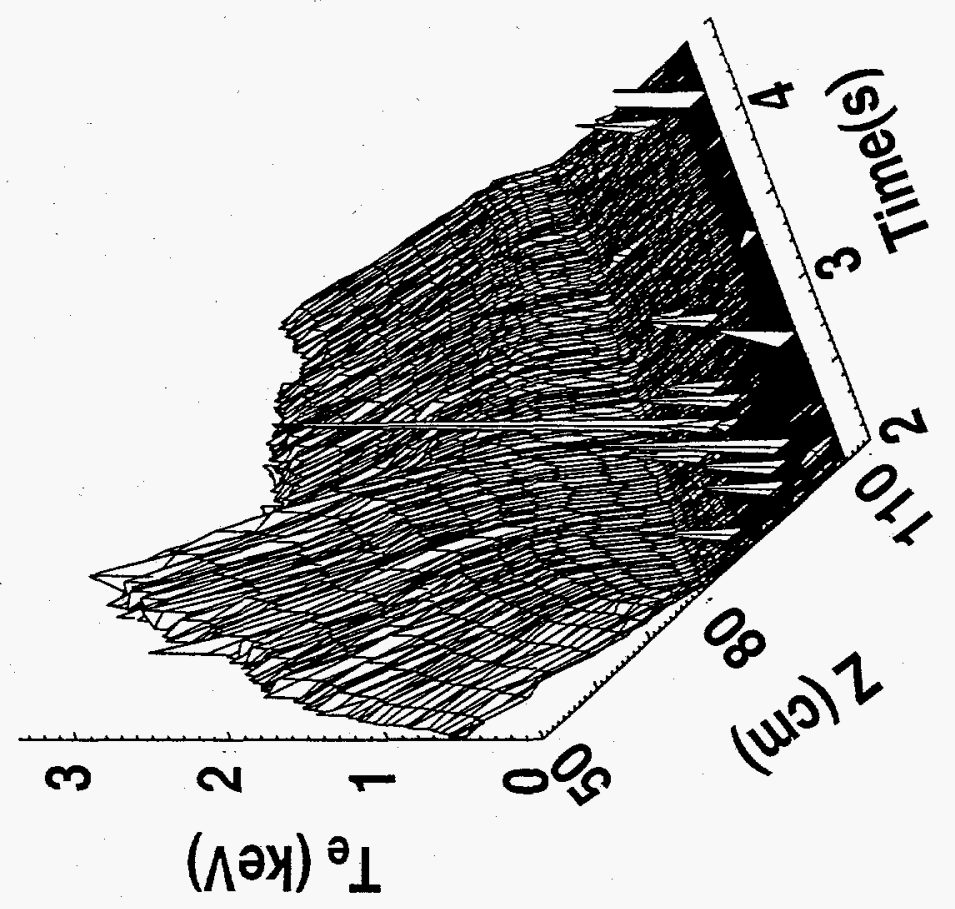

$\infty$
$\infty$
$\dot{1} I_{1}$

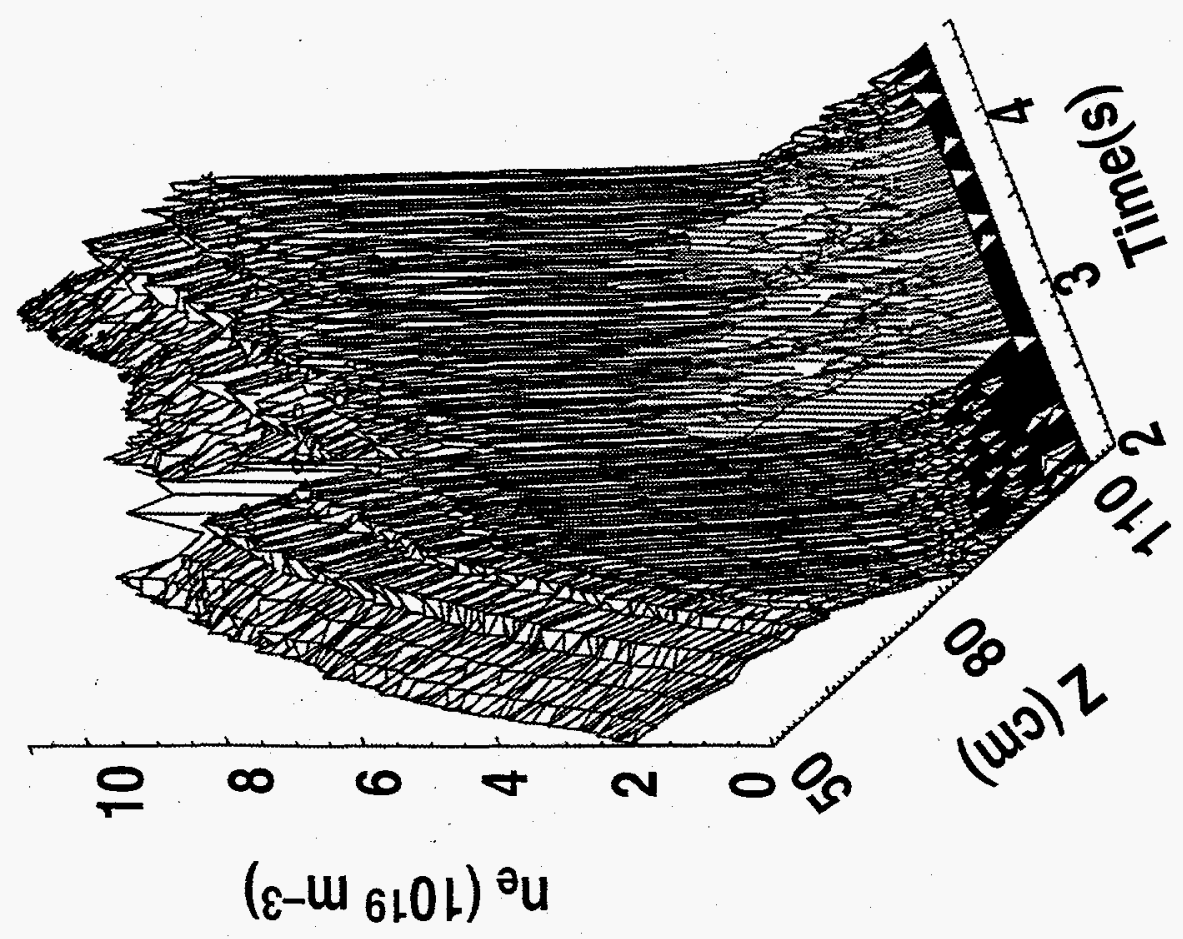




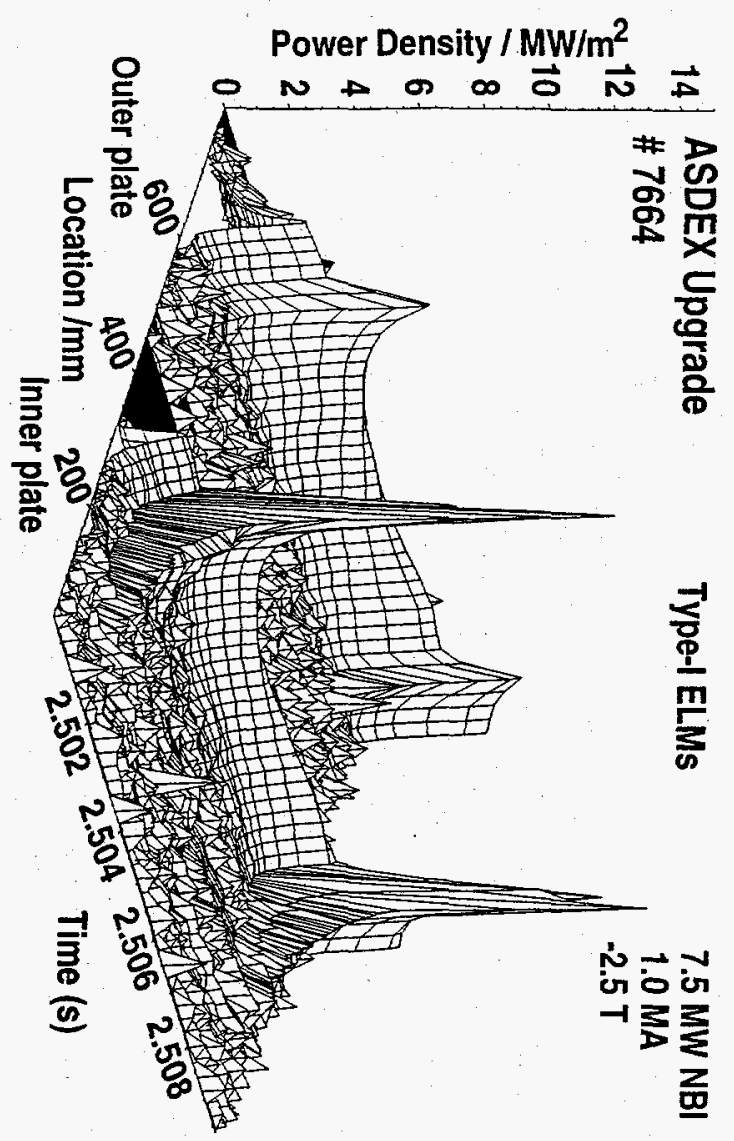

טִ̣

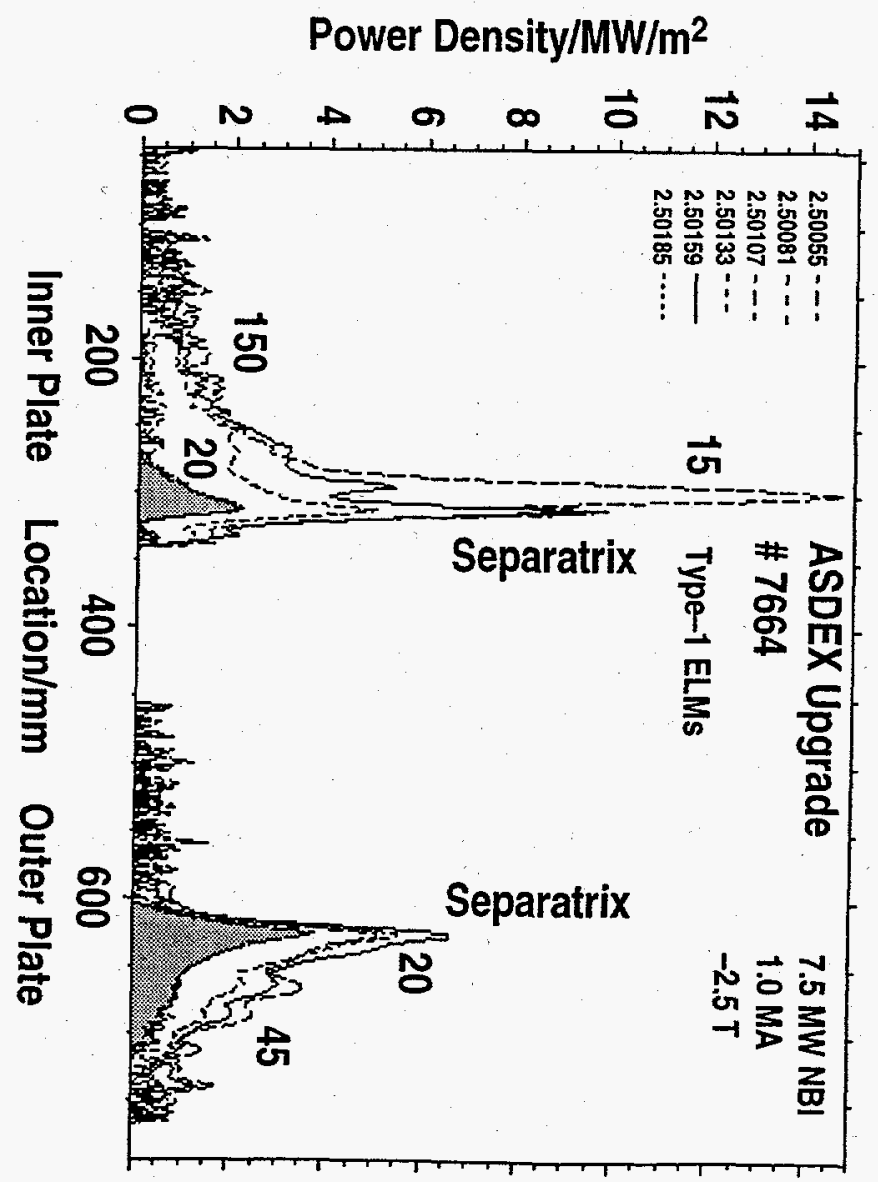




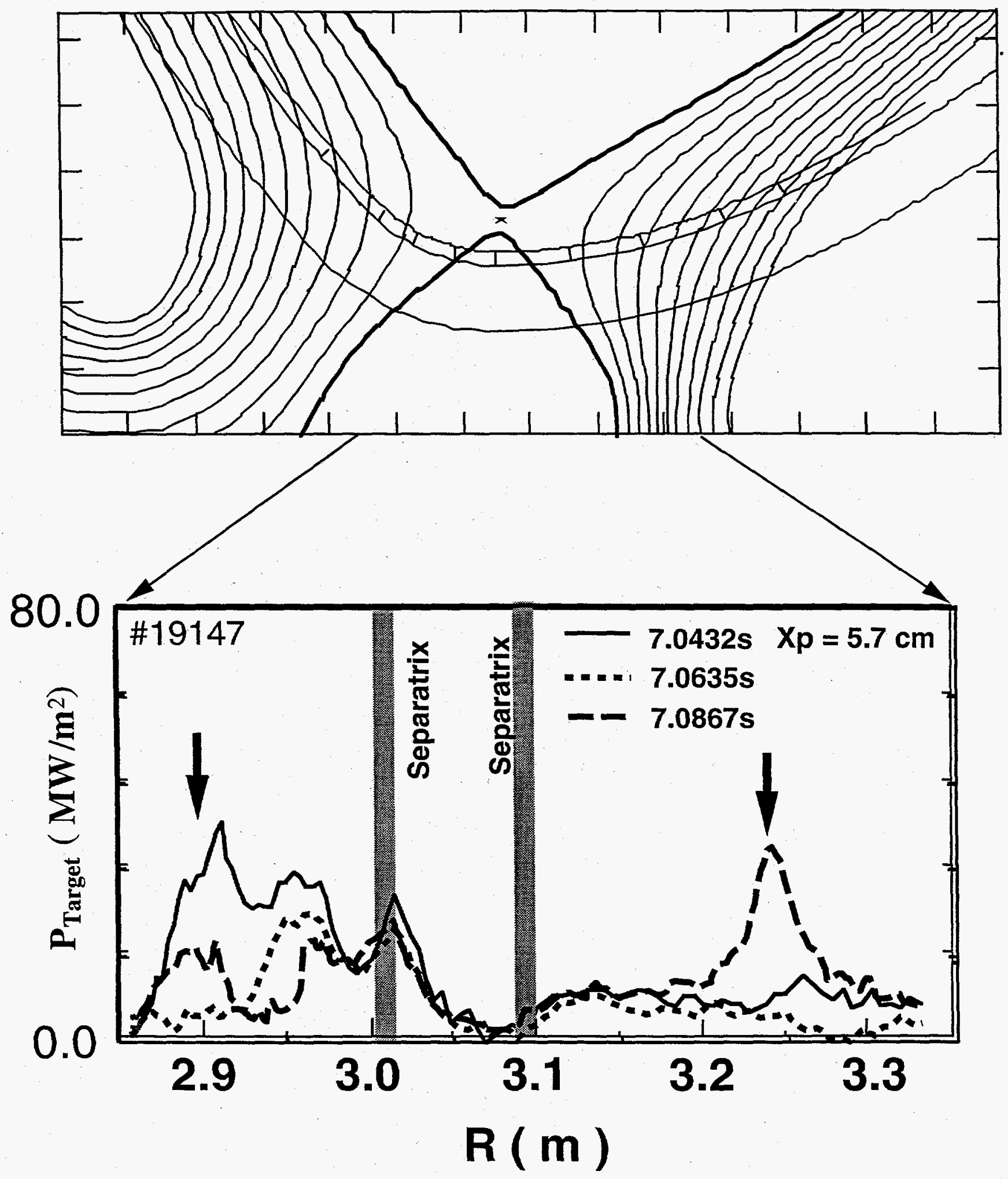

Fig. 10 


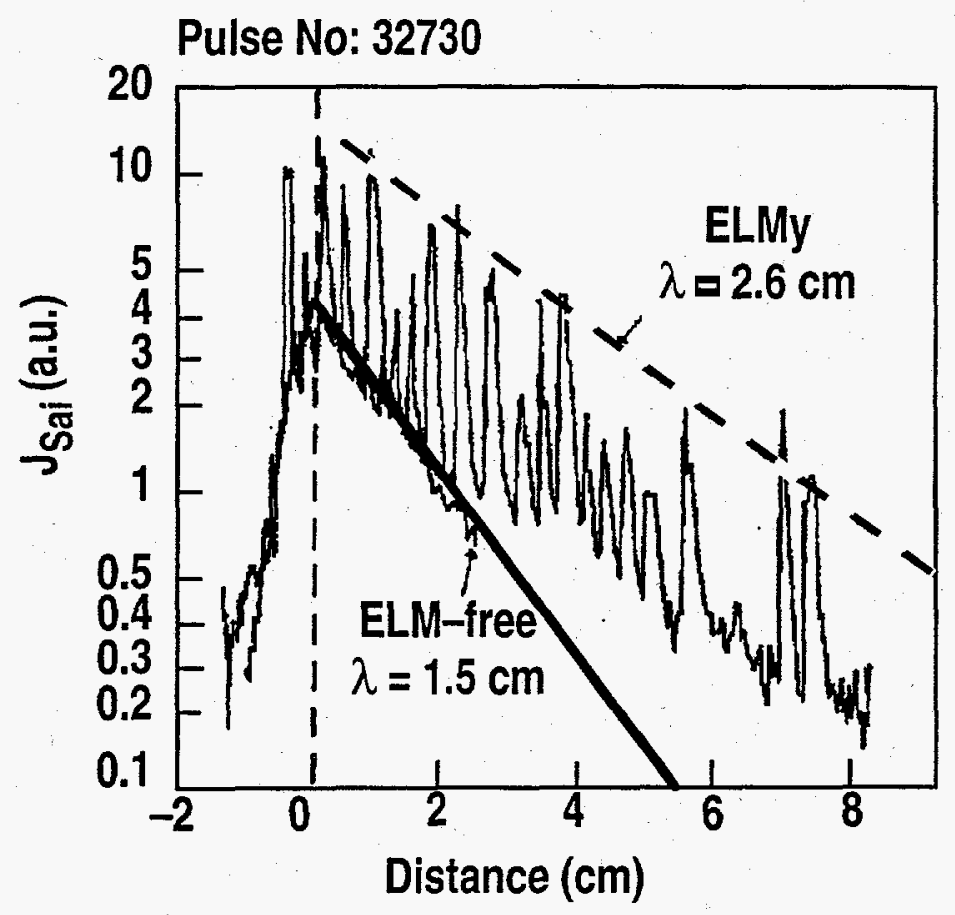

Fig. 11 


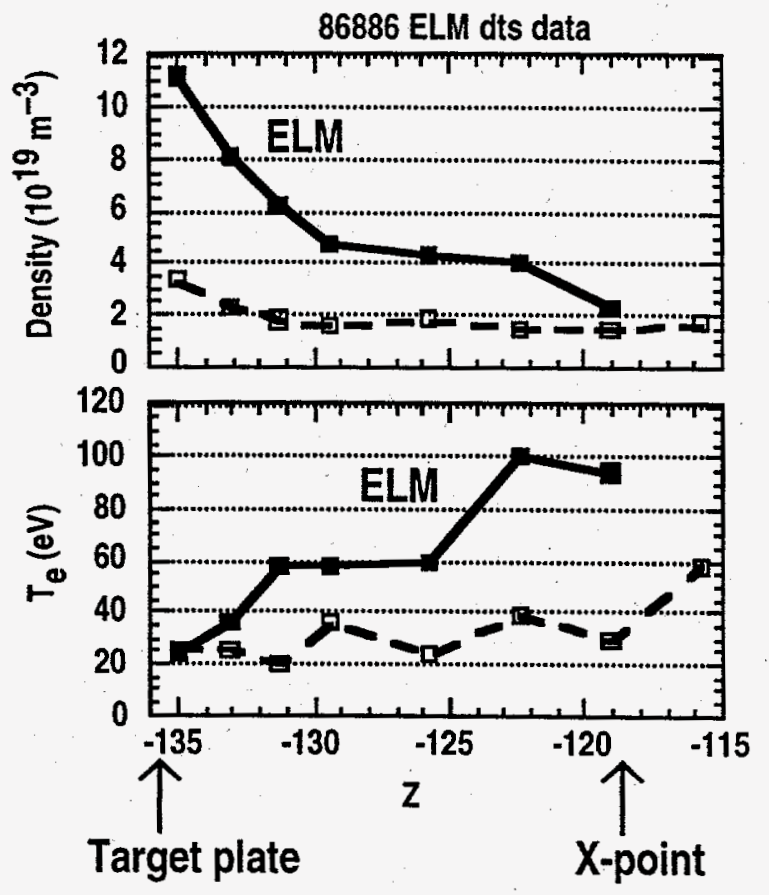

Fig. 12 


\section{ELM/DISRUPTION LIFETIME CARBON}

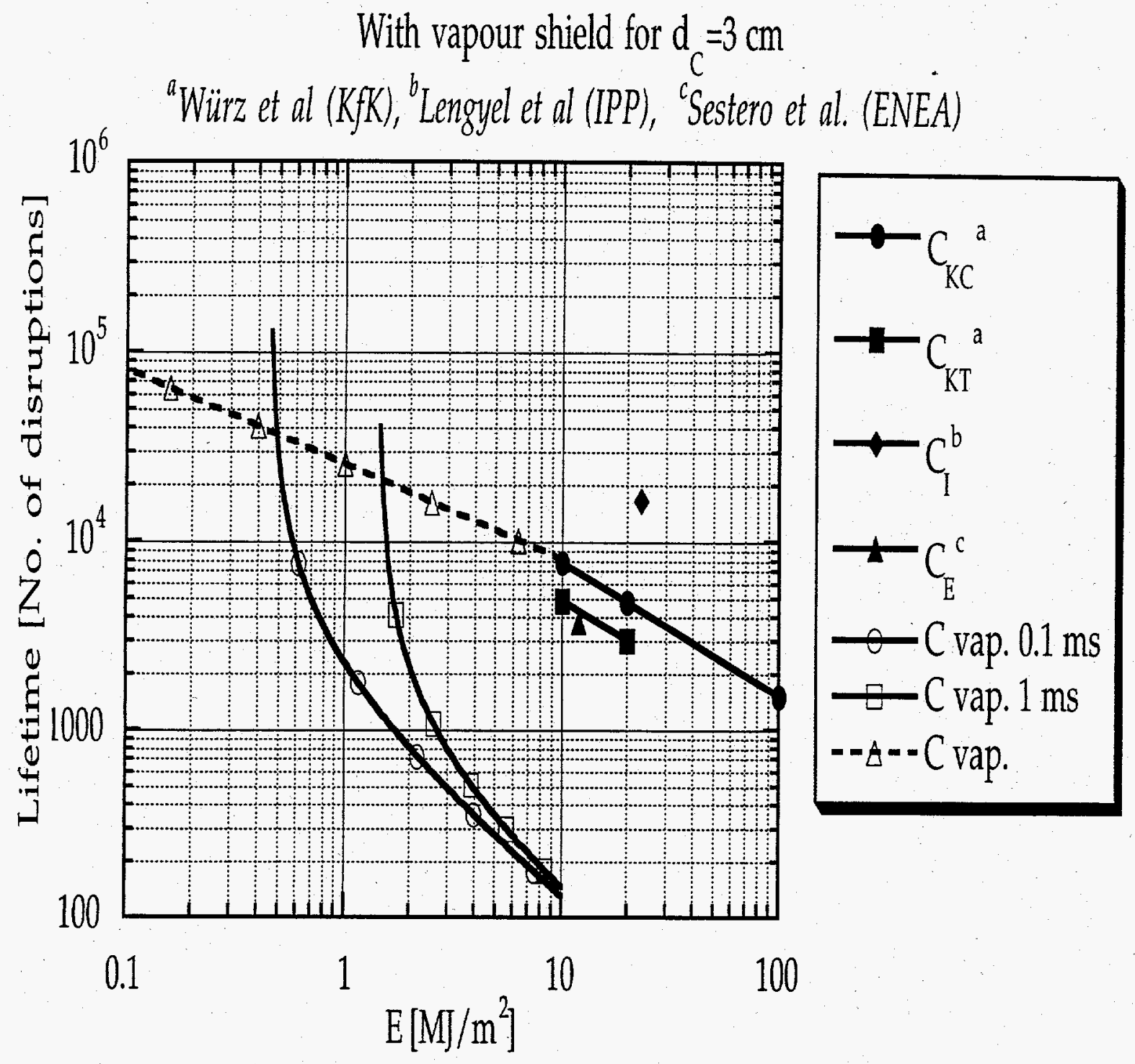

Fig. 13 

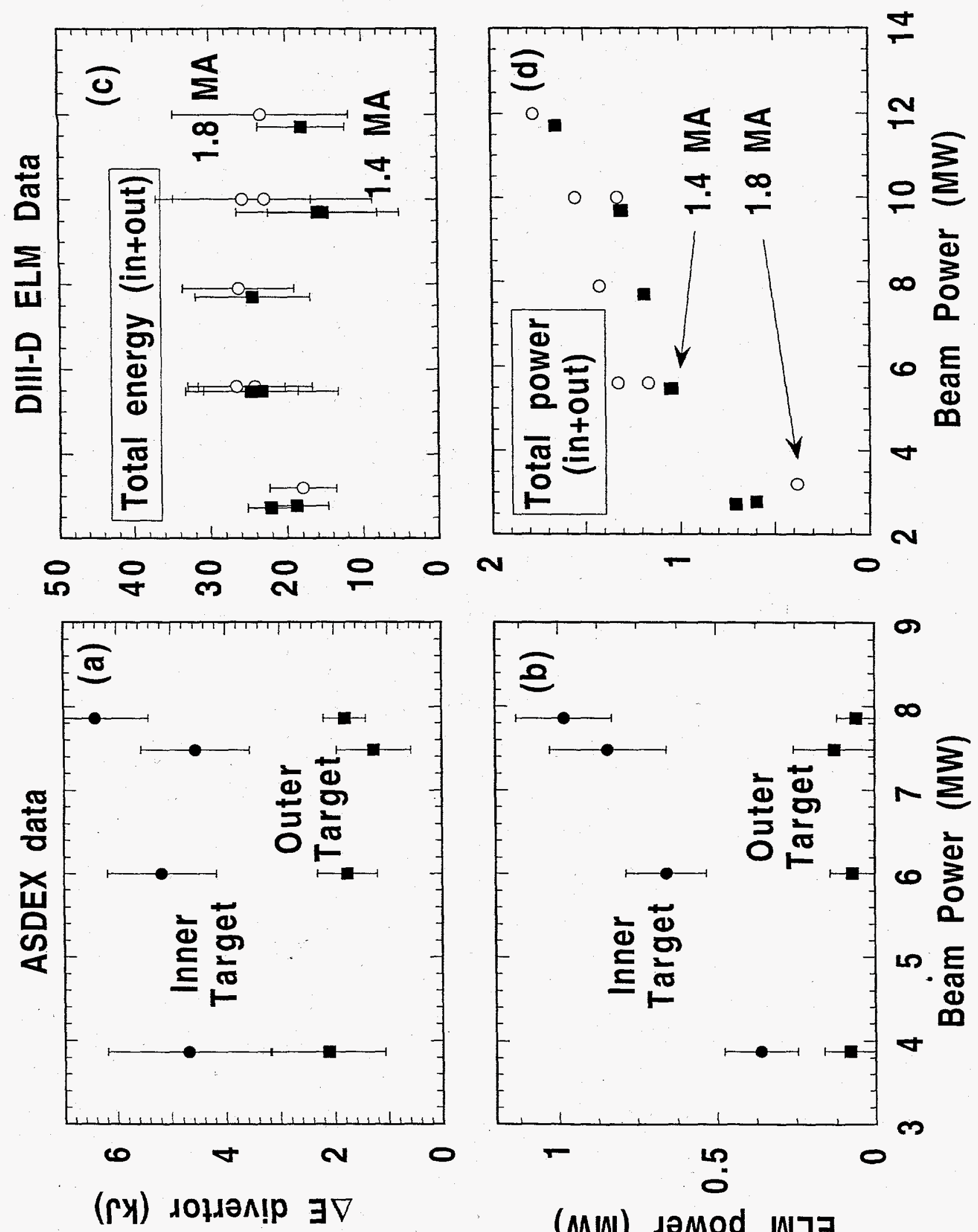

(MW) มәмоd พาษ 


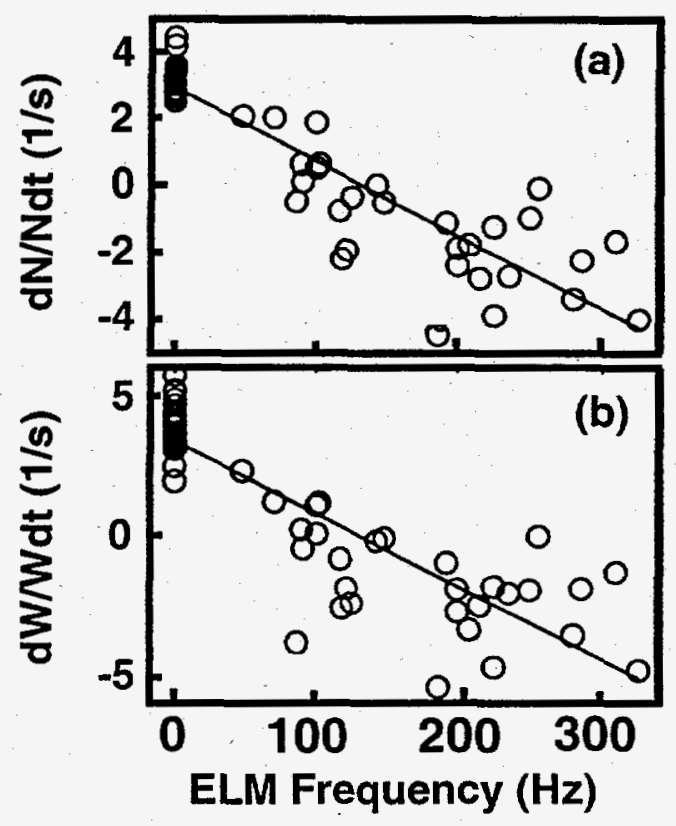

Fig. 15 


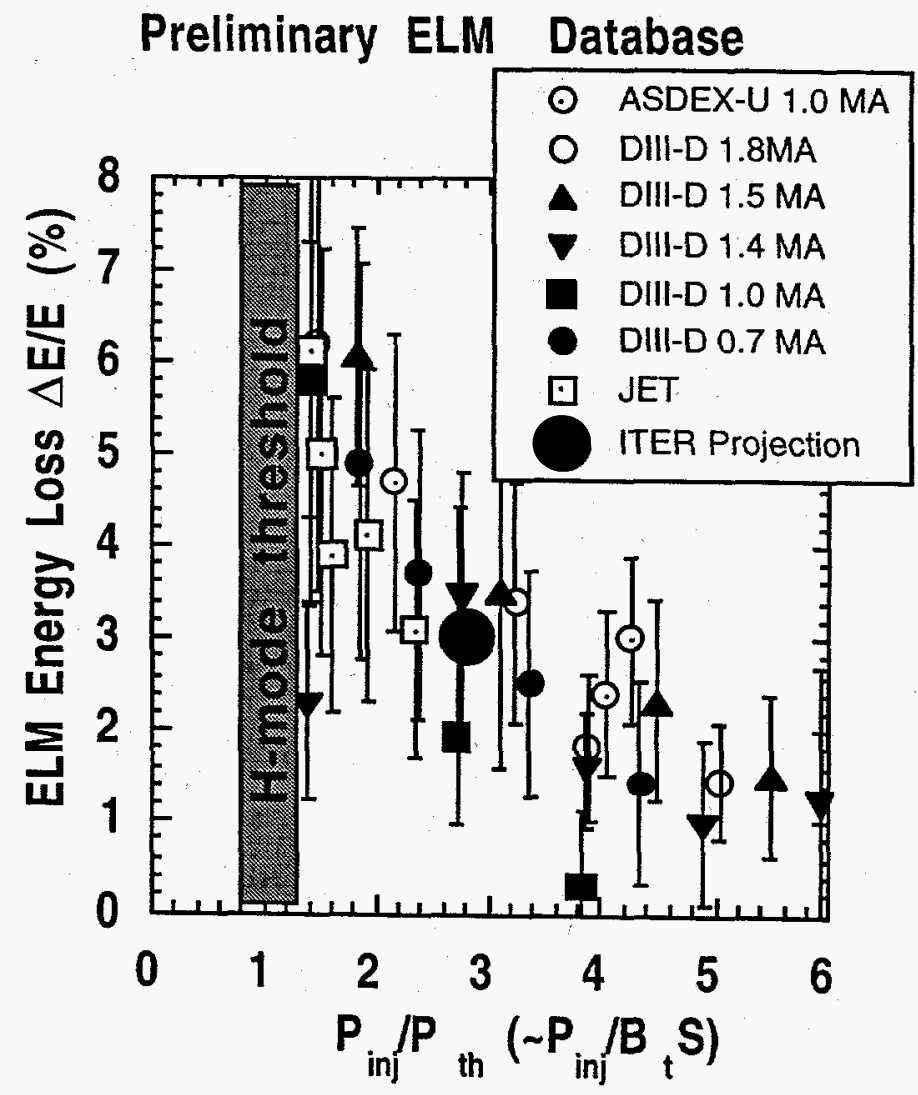

Fig. 16 


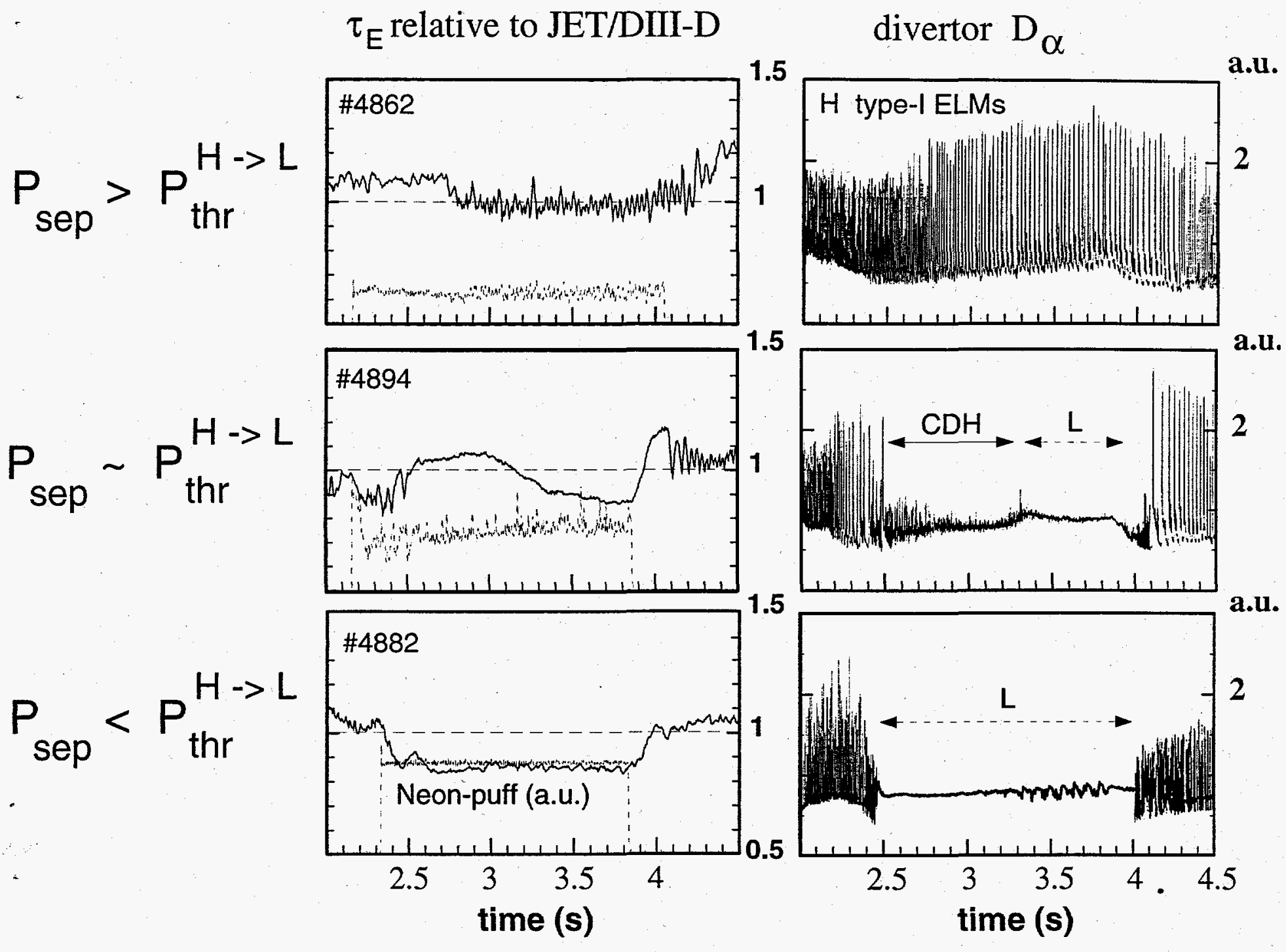

Fig. 17 


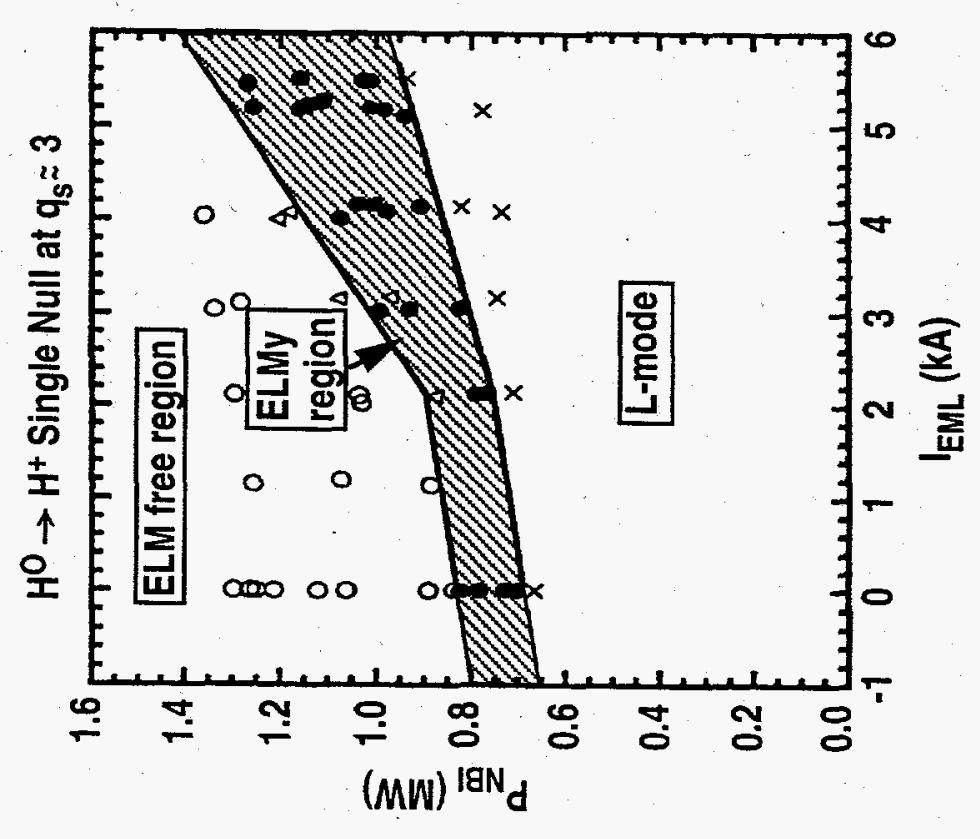

$\stackrel{\infty}{\rightarrow \infty}$ 


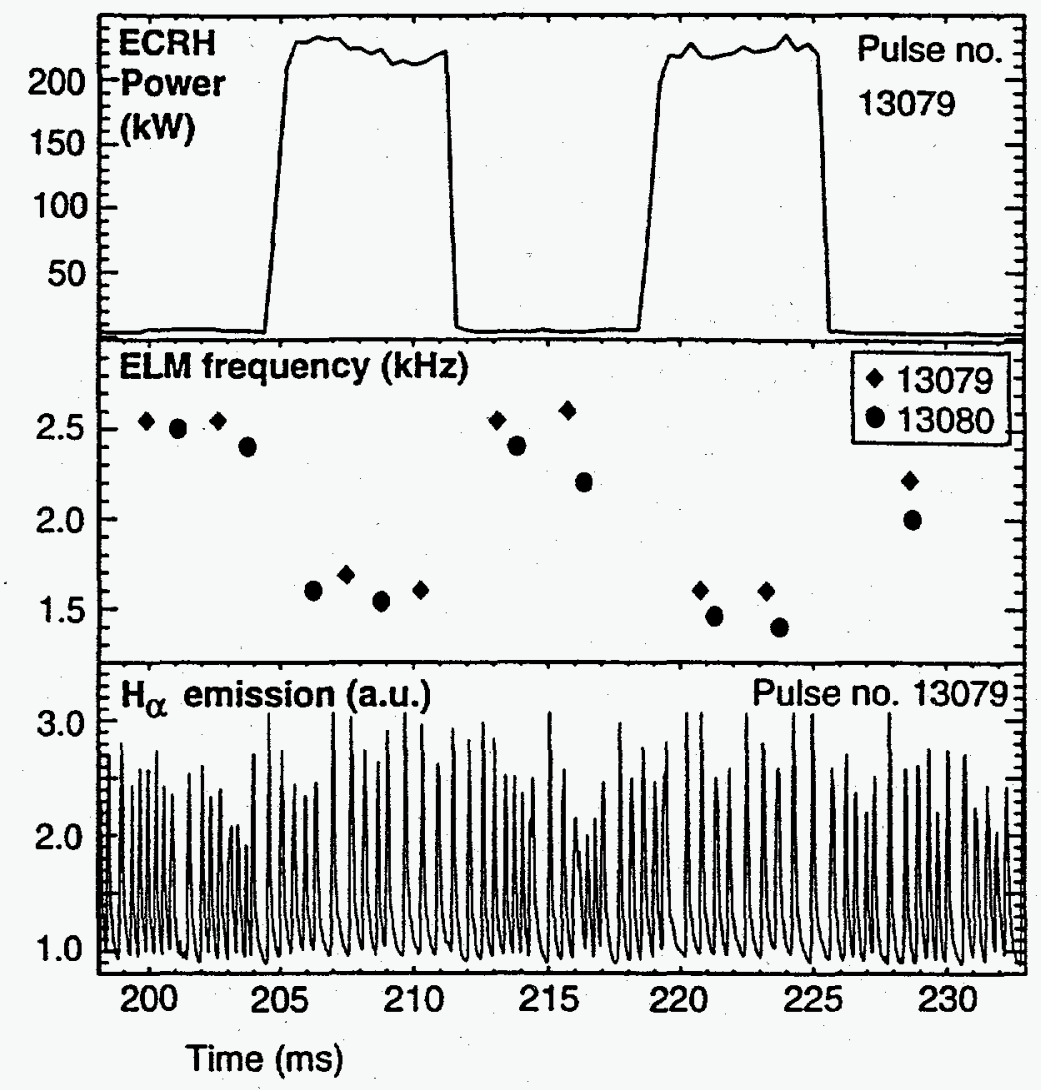

Fig. 19 
.

. 